\title{
Chemical induction of gut $\beta$-like-cells by combined FoxO1/Notch inhibition as a glucose-lowering treatment for diabetes
}

Takumi Kitamoto ${ }^{1,2}$, Yun-Kyoung Lee ${ }^{3}$, Wendy M. McKimpson ${ }^{1,2}$, Hitoshi Watanabe ${ }^{1,2}$, Nishat Sultana ${ }^{5}$, Wen $\mathrm{Du}^{1,2}$, Jason Fan ${ }^{6}$, Bryan Diaz ${ }^{5}$, Hua V. Lin ${ }^{4}$, Rudolph L. Leibel ${ }^{5}$, Sandro Belvedere ${ }^{3}$, Domenico Accili1, ${ }^{1,2}$

${ }^{1}$ Department of Medicine and ${ }^{2}$ Naomi Berrie Diabetes Center, ${ }^{5}$ Department of Pediatrics Vagelos College of Physicians and Surgeons, Columbia University, New York, NY 10032;

${ }^{3}$ Forkhead BioTherapeutics, Inc., New York, NY; and

${ }^{4}$ BioFront Therapeutics, Beijing, China

${ }^{6}$ Department of Ophthalmology, Bascom Palmer Eye Institute, University of Miami, Miami, FL, 33146

Running title: FoxO1 inhibitors in murine diabetes

Corresponding Author: Takumi Kitamoto, MD, PhD

Department of Medicine, Vagelos College of Physicians and Surgeons, Columbia University, New York, NY

Phone: $+1-2128515332$

Fax: $+1-2128515331$

E-mail: tk2752@cumc.columbia.edu

Reprint requests: Domenico Accili (da230@cumc.columbia.edu) 
Word count: 3211 words, Abstract 143 words

Total number of figures: 6

Supplementary figures: 5

\section{Highlights}

- Gut FoxO1 ablation enriched enteroendocrine cells (EECs) in mice small intestine

- Gut FoxO1 ablation can reversed hyperglycemia in diabetic Akita mice

- FoxO1 ablation in Neurog3 cells more efficiently altered EEC lineage than in panintestinal epithelial cells

- Dual FoxO1/Notch inhibition synergistically boosted EEC lineage and $\beta$-like-cells

- Orally available small molecule FoxO1 inhibitor phenocopied genetic FoxO1 ablation in generating gut $\beta$-like cells

Keywords: Diabetes; Insulin; $\beta$ cell replacement; FOXO1; FoxO1 inhibitor; Notch

\section{inhibition}




\begin{abstract}
Lifelong insulin replacement remains the mainstay of type 1 diabetes treatment. Genetic FoxO1 ablation promotes enteroendocrine cell (EECs) conversion into glucose-responsive $\beta$-like cells. Here, we tested whether chemical FoxO1 inhibitors can generate $\beta$-like gut cells. Panintestinal epithelial FoxO1 ablation expanded the EEC pool, induced $\beta$-like cells, and improved glucose tolerance in Ins $2^{\text {Akita/+ }}$ mice. This genetic effect was phenocopied by small molecule FoxO1 inhibitor, Cpd10. Cpd10 induced $\beta$-like cells that released insulin in response to glucose in mouse gut organoids, and this effect was strengthened by the Notch inhibitor, DBZ. In Ins $2^{\text {Akita/+ }}$ mice, a five-day course of either Cpd10 or DBZ induced insulin-immunoreactive $\beta$ like cells in the gut, lowered glycemia, and increased plasma insulin levels without apparent adverse effects. These results provide proof of principle of gut cell conversion into $\beta$-like cells by a small molecule FoxO1 inhibitor, paving the way for clinical applications.
\end{abstract}




\section{INTRODUCTION}

Efforts to generate insulin-producing cells that can be delivered to patients in vivo to treat type 1 diabetes have been underway for decades. Decisive progress has been made in the generation of pancreatic hormone-producing cells from embryonic stem (ES) or induced pluripotent stem (iPS) cells ${ }^{1}$. These strategies can provide an unlimited source of $\beta$-cells to address the scarcity of islet donors. However, there are at least two significant hurdles for cellbased replacement therapies: physical limitations in delivering sufficient quantities of cells, and protection from autoimmunity to maintain their function ${ }^{2}$.

An alternative approach entails conversion of endogenous non- $\beta$ cells into insulin-secreting cells ${ }^{3}$. However, this approach suffers from poor reproducibility of claims. We and others have shown that gut epithelial cells can be reprogrammed into functional glucose-sensing $\beta$-like cells 4-8. Genetic ablation of FoxO1 in EECs in vivo gives rise to $\beta$-like cells that produce and secrete insulin in a manner similar to endogenous $\beta$-cells ${ }^{6}$. In addition, FoxO1 inhibition by shRNA or a dominant-negative mutant converts cells in human iPS cell-derived gut organoids into pancreatic $\beta$-like cells ${ }^{7}$, suggesting that the ability of EECs to become insulin-producing cells is a shared property of rodent and human gut cells. This approach provides an attractive alternative strategy by which to replace $\beta$-cell mass in type 1 diabetes. In addition, gut cells can potentially escape autoimmune attack owing to their rapid turnover, scattered distribution, and immune privilege ${ }^{9}$.

To translate this interesting biology into a treatment, it is necessary to inhibit FoxO1 pharmacologically. As a member of the forkhead DNA binding domain family ${ }^{10-12}$, FoxO1 lacks a ligand binding domain, complicating the search for an appropriate small molecule agonist or antagonist. However, we have identified at least two classes of highly specific ${ }^{13}$ and selective FoxO1 inhibitors derived from an unbiased chemical library screen ${ }^{14}$. In the present study, we 
asked whether chemical FoxO1 inhibition in vivo can phenocopy the effects of somatic ablation of FoxO1 in gut epithelial cells on EEC differentiation. In addition, in light of previous findings indicating that the FoxO and Notch pathways cooperate on pancreatic $\beta$-cell development ${ }^{15,16}$, and of the effect of Notch on this process ${ }^{17}$, we also examined the effect of combining inhibitors of the two pathways on EEC differentiation and diabetes treatment. Our findings establish the feasibility of delivering a small molecule FoxO1 inhibitor to generate $\beta$-like cells in the gut and lower glycemia in insulin-deficient mice. 


\section{RESULTS}

\section{Gut FoxO1 ablation expands the EEC progenitor pool}

To investigate the mechanism by which FoxO1 ablation affects EEC formation, we generated ACTB-tdTomato-EGFP; Neurog3-Cre:FoxO $1^{f l f l}$ mice and assessed EEC number, localization, and gene expression patterns. The reporter allele yields green fluorescence after cre recombination, and allows to monitor Neurog3 expression by comparing $\mathrm{GFP}^{+}\left(\mathrm{Neurog}^{+}\right)$and $\mathrm{RFP}^{+}\left(\mathrm{Neurog}^{-}\right)$cells. In wild-type animals (ACTB-tdTomato-EGFP; Neurog3-Cre, hereafter TG-WT), EECs were located throughout the length of the villi. Following FoxO1 ablation in EEC progenitors (ACTB-tdTomato-EGFP; Neurog3-Cre (+): FoxO1 ${ }^{\mathrm{fl} / \mathrm{fl}}$, hereafter TG-NFKO), we saw an enrichment of EECs in crypts (Fig. 1A and B). Both $\mathrm{GFP}^{+}$and $\mathrm{RFP}^{+}$cells were readily identified by fluorescence-activated cell sorting (FACS) (P4 and P5 in Fig. 1C); however, $\mathrm{GFP}^{+}$cells appeared to segregate in two different subsets that differed by the strength of the $\mathrm{RFP}^{+}$signal (P5 and P6 in Fig. 1c). mRNA measurements indicated that the three subsets corresponded to $\mathrm{RFP}^{+} \mathrm{GFP}^{-}$endocrine progenitors (FoxO1 ${ }^{\text {high }}$, Tph $1^{\text {low }}$, $\mathrm{ChgA}^{\text {low }}$, $\mathrm{GFP}^{+} \mathrm{RFP}^{-}$ terminally differentiated EECs $\left(\right.$ FoxO1 ${ }^{\text {low }}$, Tph $\left.1^{\text {high }}, \mathrm{ChgA}^{\text {high }}\right)$ and $\mathrm{RFP}^{+} \mathrm{GFP}^{+}$cells (FoxO1 $\left.{ }^{\text {medium }}, \mathrm{Tph}^{\text {medium }}, \mathrm{ChgA}^{\text {medium}}\right)$ that likely represent different stages of EEC maturation from early (P5) to late progenitors (P6) (Fig. 1D-F).

Notch inhibition has been shown to expand the EEC pool ${ }^{18}$. Thus, we compared the combined effect of FoxO1 ablation and Notch blockade by the $\gamma$-secretase inhibitor, DBZ, on the different stages of the EEC lineage (Fig. 1I-H) ${ }^{16,19}$. As reported, 96-hr after treatment with a single dose of DBZ, there was a substantial increase of serotonin (5HT), somatostatin (SS), and glucagon-like peptide-1 (GLP-1) cells in C57/BL6 mice (Fig. S1A-C) ${ }^{18}$. We next compared the effect DBZ on FAC-sorted P5 and P6 cells isolated from WT or TG-NFKO mice. In contrast to 
early progenitors P5 (Fig. 1G), FoxO1 knockout increased the number of late progenitors P6 by $30 \%(0.79 \pm 0.07$ vs. $1.12 \pm 0.11: p=0.01)$ at baseline, and increased EEC number by four-fold following DBZ treatment; this number more than doubled in TG-NFKO compared to TG-WT $(4.39 \pm 1.11$ vs. $10.17 \pm 2.13: p=0.014)($ Fig. $1 \mathrm{H}-\mathrm{J})$. We also assessed EEC localization following 96-hr of DBZ treatment (Fig. 1K-L). In contrast to pre-treatment, the number of GFP cells in the crypts increased in both control and TG-NFKO mice, while more EECs were detected in the villi of TG-NFKO mice. Thus, Neurog3-driven FoxO1 ablation and Notch inhibition synergistically expanded the EEC progenitor pool and increased EEC number throughout the villi.

\section{Time course of EEC differentiation after FoxO1 ablation}

To test whether the timing of FoxO1 ablation affects the EEC pool, we ablated FoxO1 at the EEC progenitor stage in Neurog $3^{+}$cells ${ }^{20}$ or at the epithelial progenitor stage in villin ${ }^{+}$cells ${ }^{21}$. We isolated crypts from Neurog3-Cre $(+)$; FoxO1 ${ }^{\mathrm{fl} / \mathrm{fl}}(\mathrm{NFKO})$ or Villin-Cre $(+)$; FoxO1 $1^{\mathrm{fl} / \mathrm{fl}}$ (VFKO) mice to prepare primary organoid cultures (mGO) and assessed the time course of FoxO1 and EEC marker expression after DBZ treatment in vitro. FoxO1 expression decreased in WT mGO with EEC differentiation (Fig. 2A), while Neurog3 expression increased at 24-hr; ChgA and Tph1 as increased at 72-hr and peaked at 96-hr (Fig. 2B-D). Consistent with in vivo findings, DBZ increased levels of Neurog3, ChgA, and Tph1 in VFKO and NFKO to a similar extent. However, NFKO mGO showed a more time-restricted effect on Neurog3 expression than VFKO (Fig. 2B), while the effect on ChgA and Tph1 was similar (Fig. 2C, D).

We have shown previously that FoxO1 inhibition increases the number of 5HT cells in human gut organoids (hGO) ${ }^{7}$. Therefore, we assessed FoxO1- and 5HT-immunoreactive cells 
during DBZ treatment (Fig. 2E). FoxO1 was expressed in nearly all cells at the beginning of treatment, but levels were suppressed as early as 24-hr especially in crypts, and nearly abolished by 72-hr. In contrast, 5HT cells were increased at 72-hr. In VFKO mGO, 5HT cells increased as early as 48-hr and peaked at 72-hr during DBZ treatment. DBZ treatment increased the number of $\mathrm{GLP}-1^{+}$cell number in $\mathrm{mGO}$ isolated from wild type mouse ileum and, again consistent with findings in $\mathrm{hGO}^{7}$, we found a higher number of GLP-1 ${ }^{+}$cells in VFKO $\mathrm{mGO}$ at baseline and after DBZ treatment compared to control (Fig. 2F). We next tested the effect of dual inhibition of FoxO1 and Notch on the generation of $\beta$-like-cells. We detected C-peptide ${ }^{+}$cells in DBZ-treated VFKO mGO after 96-hr (Fig. 2G). These results suggest that dual inhibition of FoxO1 and Notch enhanced the number of EEC lineage cells, including GLP-1 ${ }^{+}$cells, and re-directed a subset of cells toward the $\beta$-like-cell phenotype.

\section{Metabolic impact of pan-gut epithelial FoxO1 ablation}

We have shown that Neurog3-driven FoxO1 ablation lowers glycemia through effects conveyed by gut $\beta$-like-cells in mice rendered diabetic with streptozotocin (STZ) ${ }^{6}$. To examine the impact of pan-epithelial gut FoxO1 ablation on glucose metabolism, we analyzed VFKO mice by immunohistochemistry and oral (OGTT) or intraperitoneal (ipGTT) glucose tolerance tests. Consistent with the mGO experiments, we found increased numbers of GLP- $1^{+}$cells in ileum and $\mathrm{GIP}^{+}$cells in duodenum compared to controls (Fig3A, B). We found C-peptide ${ }^{+}$cells exclusively in VFKO mice (Fig. 3C). VFKO mice showed a slight decrease of glucose AUC during OGTT (Fig. 3 D and F), but not ipGTT (Fig. 3E and G). Plasma insulin concentrations were significantly higher at 15 and 30 min during OGTT in VFKO compared to controls, presumably because of the gut insulin ${ }^{+}$cells and increased GLP-1 ${ }^{+}$cells (Fig. $\left.3 \mathrm{H}\right)$. 


\section{Gut FoxO1 ablation reverses hyperglycemia in INS2 ${ }^{\text {Akita/+ }}$}

To investigate the glucose-lowering effect associated with induced intestinal $\beta$-like cells, we introduced null FoxOl alleles in an insulin-deficient diabetic mouse model. Akita mice develop insulin-dependent diabetes by 3 - to 4 -weeks of age due to a dominant-negative mutation in the Ins 2 gene $^{22}$. We crossed VFKO mice with INS2 ${ }^{\text {Akita/+ }}$ mice to generate INS2 ${ }^{\text {Akita/+ }}$; Vil-Cre (+): FoxO1 ${ }^{\mathrm{fl} / \mathrm{fl}}$ (Akita-VFKO) and WT littermate controls (INS2 ${ }^{\text {Akita/++}} ; \mathrm{FoxO}^{\mathrm{fl} / \mathrm{fl}}$ (Akita-WT). We monitored glucose levels from 4 to 24 weeks in ad libitum-fed conditions. Both Akita-WT and Akita-VFKO mice showed increased glucose levels starting at 4 weeks, but Akita-VFKO mice showed only mild hyperglycemia and maintained consistently lower glucose level than AkitaWT for the duration of the experiment (Fig. 4A). This effect was confirmed after 4-hr fasting and was not associated with hypoglycemia (Fig. 4B). Plasma insulin levels in ad libitum-fed AkitaVFKO mice were significantly higher compared to Akita-WT starting at 10 weeks of age (Fig. 4C). Moreover, OGTT in 12-week-old Akita-VFKO animals demonstrated significant improvement in glucose tolerance compared to Akita-WT (Fig. 4D). To evaluate whether the improved glucose tolerance was due to increased numbers of GLP-1 cells, we performed OGTT in the presence of the GLP-1 antagonist, exendin-9. Exendin-9 treatment alone increased glucose levels in both groups, but did not affect the improvement observed in Akita-VFKO mice, indicating that it is GLP-1-independent (Fig 4E, F).

GIP-, GLP-1-, and C-peptide-immunoreactive cells in duodenum and ileum increased significantly in Akita-VFKO compared to controls (Fig. 4G-I). These data are consistent with the glucose-lowering effect of gut-derived $\beta$-like cells observed in STZ-treated Neurog3-FoxO1 mice ${ }^{6}$. 


\section{Induction of cell conversion by FoxO1 and Notch inhibition}

Next, we asked whether a previously described selective FoxO1 inhibitor (compound 10; hereafter, Cpd 10) ${ }^{13,14}$ can induce $\beta$-like cells in gut organoids and in vivo. In primary mGO cultures, Cpd 10 phenocopied the effect of genetic FoxO1 ablation on EEC differentiation, resulting in a time-dependent induction of Neurog3, ChgA, and Tph1 compared to vehicle (Fig. 5A-D). Interestingly, Cpd10 also increased FoxO1 mRNA levels, consistent with the effect of dominant-negative FoxO1 mutants to cause a compensatory activation of endogenous Foxol transcription ${ }^{7}$. These gene expression changes were confirmed by immunohistochemistry with FoxO1 and Tph1 antibodies (Fig. S2A). Cpd10 treatment induced C-peptide-immunoreactive cells but -unlike the genetic ablation- did not induce GLP-1 cells (Fig. S2B, C). To assess the function of these cells, we compared GLP-1 and insulin secretion in mGO from VFKO and NFKO mice, or following pharmacological inhibition with Cpd10 in WT mice. Organoids from VFKO mice showed increased basal and glucose-stimulated GLP-1 secretion compared to WT (Fig. 5E). Treatment with DBZ increased basal secretion in control mGO to the levels seen in VFKO and further raised glucose-dependent GLP-1 secretion (Fig. 5E). In contrast, Cpd10 had no effect on GLP-1 secretion in WT mGO, whereas DBZ increased both basal and glucosestimulated GLP-1 (Fig. 5F). These data are consistent with the immunohistochemistry showing no effect of Cpd10 on the number of GLP-1 ${ }^{+}$cells (Fig. S2B).

mGO derived from NFKO or VFKO mice did not show detectable insulin secretion in response to glucose, likely due to the small number of $\beta$-like cells. However, addition of DBZ to the medium resulted in robust glucose-stimulated insulin secretion, $\sim 2.6$-fold higher in NFKO than VFKO mGO (Fig. 5G). In WT mGO, Cpd10 alone induced glucose-dependent insulin 
secretion, and this effect was enhanced by DBZ treatment (Fig. 5H, Fig. S2C). Thus, whereas

DBZ has an independent effect to increase GLP-1 secretion ${ }^{23}$, the augmented insulin secretion effect of DBZ requires either genetic or pharmacologic inhibition of FoxO1.

\section{FoxO1 and Notch inhibitors lower glucose in INS2 ${ }^{\text {Akita/+ }}$}

To evaluate the synergistic effects of FoxO1 and Notch inhibition in the gut in vivo, we administered Cpd10 by intraperitoneal injection with or without oral dosing with the notch inhibitor PF-03084014 (hereafter, PF) ${ }^{24}$ to INS2 $2^{\text {Akita/+ }}$ mice for 5 days. In wild type mice, Cpd10 concentrations in the duodenum peaked within 1-hr of administration compared to plasma and dropped into the low nM range by $24-\mathrm{hr}$ (Fig. S3A). At the employed dose (50mg/kg), Cpd10 did not affect hepatic G6pc or Pckl expression (Fig. S3B). We optimized dosing and found that oral dosing at $150 \mathrm{mg} / \mathrm{kg}$ increased EEC number and retained proliferative cells in crypts (Fig S4A-E). Combination treatment with $50 \mathrm{mg} / \mathrm{kg}$ of Cpd10 and $150 \mathrm{mg} / \mathrm{kg}$ of PF yielded the highest number of EECs and induced $\beta$-cell marker expression (Fig. S4F-G).

Neither single nor combination therapy affected body weight (Fig. 6A). Ad lib-fed glucose concentrations decreased by $\sim 150-170 \mathrm{mg} / \mathrm{dl}$ in mice treated with either Cpd10 or PF alone, and a 4-hr-fast did not induce hypoglycemia (Fig. 6C). Combination treatment had no additional benefit on mean glucose concentrations, except in a subset of mice in which glucose levels were $<200 \mathrm{mg} / \mathrm{dl}$ (Fig. 6B). However, combination treatment had an additive effect to improve ipGTT (Fig. 6C-D). Treatment with Cpd10 alone was also associated with increased insulin concentrations (Fig. 6E).

Combination treatment had an additive effect on Neurog3, ChgA, Nkx6.1, and Nkx2.2 mRNA in duodenum, whereas PF alone was sufficient to achieve maximal induction of Tph1 
mRNA (Fig. 6N). Immunohistochemistry demonstrated a synergistic effect of combination treatment to generate up to $\sim 8 \mathrm{C}$-peptide ${ }^{+}$cells/crypt and $\sim 19$ insulin $^{+}$cells per villus-crypt unit (Fig. 6F-K, 6O-P, S5A-E). 


\section{DISCUSSION}

The present study demonstrates that pharmacological inhibition of FoxO1 can induce $\beta$-like cells in the gut to a similar extent as genetic FoxO1 knockout. This remarkable property of small molecule FoxO1 inhibitors has not been previously reported. This finding expands on work showing that $\mathrm{Cpd} 10$ can reduce hepatic glucose production from pyruvate in vivo ${ }^{13}$. Successfully applied to humans with diabetes, this strategy would provide an alternative to insulin injections, circumventing the risk of hypoglycemia and eliminating the need for frequent glucose monitoring, thus significantly reducing the burden of care and potentially improving glycemic control. Whether gut-derived $\beta$-like cells would be targeted by autoimmunity remains a concern. However, based on the distinct immunity of the gut mucosa compared to the pancreas ${ }^{9}$, rapid cell turnover, and scattered distribution of $\beta$-like cells, it can be argued that they can escape functionally consequential autoimmune engagement ${ }^{25}$.

Since the original report on the role of FoxO1 in the gut ${ }^{6}$, the impact of FoxO1 ablation on trans-differentiation of entero-endocrine cells (EECs) has been confirmed in human gut organoids ${ }^{7}$. Experimental co-expression of Neurog3, Pdx1, and MafA can generate $\beta$-like cells in the gut ${ }^{5}$. FoxO1 inhibition recapitulates these transcriptional effects ${ }^{26,27}$. FoxO1 activation can suppress Neurog $3^{28}$, possibly via O-GlcNAc transferase resulting in fewer GLP-1 cells ${ }^{29}$.

\section{Mechanism of cell conversion and combination with Notch}

To define the stage in EEC differentiation affected by FoxO1 ablation, we show that FoxO1 inactivation in epithelial progenitors (VFKO) phenocopies inactivation in the EEC progenitor (NFKO), consistent with a primary role of FoxO1 in EEC progenitors to suppress the $\beta$-like-cell phenotype. We also identified two developmental stages in the EEC progenitors by dual tracing 
with GFP and RFP, and tentatively mapped the role of FoxO1 inhibition to the late progenitor/early EEC stage. This narrow window of FoxO1 action may explain why the yield of insulin-immunoreactive cells is comparatively lower in FoxO1 knockout models than in the triple transcription factor gain-of-function transgenics ${ }^{30,31}$.

Leveraging an extensive prior body of work regarding FoxO1 and Notch to regulate endocrine cell lineage ${ }^{15,32,33}$, we successfully tested a combination regimen that increased the number of $\beta$-like cells. Notch inhibition promoted expansion of the EEC lineage, truncating the stage between $\operatorname{Lgr} 5^{+}$and Neurog $3^{+}$. By expanding the pool of available late progenitors, we saw an increased generation of $\beta$-like cells. We also detected suggestive evidence of an additive glucose-lowering effect in a subset of Akita mice treated with the combination of Cpd10 and PF. Permutations of the dosing regimen (e.g., route of delivery, pulse-chasing, timed delivery, alternate day) will be needed to explore the full glucose-lowering potential of the combination.

There are differences between genetic ablation and chemical inhibition of FoxO1. For example, Villin-driven FoxO1 ablation increased both GLP-1 and $\beta$-like cells, while Cpd10 increased insulin, but not GLP-1 secretion. These differences may reflect selective inhibition of FoxO1 targets by $\mathrm{Cpd10,}$ as shown in prior work in which this compound demonstrated selective inhibition of expression of G6pc but not Gck in liver ${ }^{14}$. A similar situation may contribute to the different effects on generation of GLP-1-expressing cells. Cpd10 may specifically inhibit FoxO1 targets involved in cellular lineage specification. Development of additional FoxO1 inhibitors may enable differential targeting of the EEC lineage-promoting and $\beta$-like cell-promoting functions.

\section{Risks and limitations}


We are aware of the risks and limitations of the approaches used here. While there is growing interest in FoxO proteins as drug targets ${ }^{34}$, the gamut of their functions makes prediction of potential adverse effects daunting. Two areas of concern are carcinogenesis ${ }^{35}$ and autoimmunity 36. Inducible post-natal deletion of FoxO1, 3, and 4 resulted in thymic lymphomas and hemangiomas ${ }^{35}$. However, this phenotype requires inactivation of all three isoforms, while our inhibitors are restricted to FoxO1, a counter-screen against FoxO3 being part of the developmental pipeline ${ }^{13,14}$. Furthermore, evidence linking FoxO1 loss-of-function to cancer is sparse and contradictory, while somatic FoxO1 gain-of-function is associated with forms of nonHodgkin lymphoma ${ }^{37,38}$ suggesting that FoxO1 inhibitors could indeed be beneficial in some cancers. Furthermore, FoxO1 is not expressed in gut epithelial stem cells, and inhibitors can be designed to provide minimal systemic exposure, further lowering the risk. We did not see gut neoplasia in VFKO mice of up to 24 weeks of age. Integrating these insights, gut-restricted FoxO1 inhibition is unlikely to incur carcinogenic risks.

Another potential adverse effect of FoxO1 inhibition is autoimmunity, which would be problematic in type 1 diabetes ${ }^{36}$. Evidence of FoxO1 deletion causing autoimmunity comes exclusively from genetic knockouts in highly selective cell types, such as Treg cells ${ }^{36}$. Unlike the widely agreed metabolic functions of FoxO1, the immune functions of FoxO1 are highly context-dependent, including evidence of protecting against autoimmunity in gut and airway 39,40. Given the multiplicity of roles assigned to FoxO1, and the apparent safety of gut inhibition, future treatments should aim for a gut-restricted compound with minimal systemic exposure.

Notch inhibitors have been tested in Alzheimer's disease and cancer, generally with modest efficacy but excellent safety profiles; modest gastrointestinal upset due presumably to goblet cell hyperplasia has been reported ${ }^{41}$. Whether the combination with FoxO1 inhibitors will increase 
such effects remains to be seen. However, given the turnover rate of the gut epithelium, any gastrointestinal adverse effects are expected to be rapidly reversible after cessation of treatment, and this would be formally assessed in preclinical safety studies in due course. It should also be pointed out that a clinical development path for a type 1 diabetes indication is only marginally affected by the need to test compounds alone and in combination, since clinical efficacy can be determined swiftly with a limited number of subjects ${ }^{42}$. In the present in vivo studies, we used an early generation Notch inhibitor with anti-hepatocellular carcinoma effects in mice ${ }^{24}$. The antihyperglycemic effect of dual inhibition was evident after 5 days of treatment.

The present study paves the way to develop an oral treatment to replace insulin via gutderived insulin-secreting cells for type 1 diabetes patients. While the present compound combination provides proof-of-principle, further combinations of FoxO1 and Notch inhibitors should be explored to maximize glucose-lowering effects and de-risk toxicity and safety. Success would result in a paradigm-shifting strategy to replace insulin administration in type 1 diabetes. 


\section{Online Methods}

\section{Animals}

Mice were housed in a climate-controlled room on a $12 \mathrm{~h}$ light/dark cycle with lights on at 07:00 and off at 19:00, and were fed standard chow (PicoLab rodent diet20, 5053; PurinaMills). Male mice of C57BL/6J background aged 8-12 weeks were used. To generate enteroendocrineand epithelial gut-specific FoxO1 knockouts, we crossed FoxO1lox/lox and Tg (Neurog3cre)C1Able/J (Ngn3-Cre) ${ }^{20}$ or B6.Cg-Tg(Vil1-cre)1000Gum/J (Vil-Cre) ${ }^{21}$ transgenic mice. For lineage tracing and cell sorting, we crossed Gt(ROSA)26Sortm4(ACTB-tdTomato,-EGFP)Luo/J $(\mathrm{mTmG})^{43}$ with Ngn3-Cre mice. For diabetic mice model, we used C57BL/6-Ins2Akita/J (Akita) 22. To assess the time course of blood glucose levels with/without FoxO1 knockouts in Akita mice, we measured blood glucose levels between 09:00 to 10:00 in ad libitum condition. Blood glucose was measured from tail clip capillary blood using (CONTOUR®NEXT ONE, Ascensia, USA). In the Enteroendocrine induction model, we injected Gamma-secretase inhibitor (DBZ, 25 mpk dissolved in 0.5\% Methocel / 0.1\% Tween80) (Chiralix, Netherlands) at 10:00 for two consecutive days. Tissues were harvested 96-hr after the first injection and analyzed by immunohistochemistry. All animal experiments were in accordance with NIH guidelines, approved and overseen by the Columbia University Institutional Animal Care and Use Committee. 


\section{Metabolic testing}

We used male mice aged 8-12 weeks. We performed intraperitoneal or oral glucose tolerance tests after a $4 \mathrm{~h}-(10 \mathrm{a} . \mathrm{m}$. to 2 p.m. $)$ or $16 \mathrm{~h}-(6 \mathrm{p} . \mathrm{m}$. to 10 a.m. $)$ fast administering 1 to 2 $\mathrm{g} / \mathrm{kg}$ glucose infusion by i.p. or p.o. Blood glucose and insulin measurements were made from tail vein blood. Insulin was measured with a mouse-specific ELISA kit (Mercodia, USA). For glucose tolerance test with pharmacological GLP-1 blockade, $10 \mu \mathrm{g} / 100 \mu \mathrm{l}$ per mouse of Exendin (9-39) (Bachem, CA) or vehicle (saline) were injected by i.p. 20 minutes before glucose measurements.

\section{Drug treatment}

6- to 7-week-old male Akita or C57BL/6J mice were acclimated for two weeks and randomized according to body weight and ad libitum glucose levels. Cpd10 was formulated in 5\% Kolliphor® HS 15 /water for i.p injection or N,N-Dimethylacetamide:Kolliphor® HS 15: water $=5: 10: 85(\mathrm{v} / \mathrm{v} / \mathrm{v})$ solution, $\mathrm{pH} 4 \sim 5$ for oral gavage injection. PF-03084014 was formulated in N,N-Dimethylacetamide:Kolliphor ${ }^{\circledR}$ HS 15: water=5:10:85 (v/v/v) solution, $\mathrm{pH} \sim 5$. Mice were dosed at $10 \mathrm{ml} / \mathrm{kg}$ twice with Cpd10 by i.p., and once with PF-03084014 daily for 5-15 days. Body weight was monitored daily in the morning before the first dose, and ad libitum blood glucose levels were monitored every 2-3 days using Contour Next glucometer or StatStrip Xpress (Novamedical) from tail vein. Oral glucose tolerance test (OGTT) was performed 5 days 
after treatment, by gavaging $2 \mathrm{~g} / \mathrm{kg}(10 \mathrm{ml} / \mathrm{kg})$ of D-glucose (Sigma) dissolved in the distilled water after 4-hr fasting from 8:30 am to 12:30 pm. Tail blood glucose was measured at 0,15, 30, 60 and $120 \mathrm{~min}$. Mice were dosed 2-hr prior to injecting glucose.

\section{Chemicals}

Ketamine was from KetaSet ${ }^{\circledR}$ and Xylazine from AnaSed ${ }^{\circledR} ; \operatorname{Ketamine}$ was from

KetaSet ${ }^{\circledR}$ and Xylazine from AnaSed ${ }^{\circledR}$; DBZ from Chiralix, PF-03084014 (Nirogacestat) from

SelleckChem; Cpd10 from ForkheadBioTherapeutics, and Kolliphor ${ }^{\circledR}$ HS 15 from

MilliporeSigma; N,N-Dimethylacetamide from MilliporeSigma. Advanced DMEM/F12, HBSS,

EDTA, HEPES were from Invitrogen; Bovine Serum Albumin (BSA) from Fisher Scientific.

16\% paraformaldehyde (PFA) was from Electron Microscopy Sciences and was diluted in sterile phosphate buffer solution to $4 \%$ final concentration.

\section{Drug exposure test}

8-week-old C57BL6/J mice were used for drug exposure experiments. Mice were fasted for $2-\mathrm{hr}$, and $10 \mathrm{mg} / \mathrm{kg}$ Cpd10 was administered by gavage. Blood was collected and small intestine harvested 1-, 4-, 8-, and 24-hr after dosing. The samples were treated with acetonitrile/methanol $(1: 1 \mathrm{v} / \mathrm{v})$, centrifuged and analyzed by LC/MS (pump: NEXERA LC30AD; controller: Shimadzu CBM-20A; autosampler: PAL System; mass spectrometer: ABSciex API4000; column: Advantage ECHELON C18 $4 \mu \mathrm{m} 20 \times 2.1 \mathrm{~mm}$ (SpriteAE1822)) 
using a gradient (phase A: Water/1M NH4HCO3, 2000:10, v/v; phase B: MeOH/1M NH4HCO3, 2000:10, v/v). Results were confirmed by biological duplicates at each time points.

\section{RNA isolation and quantitative PCR analysis}

We lysed whole gut, FAC-sorted cells $\left(5-10 \times 10^{3}\right)$, or mGO in $1 \mathrm{~mL}$ TRIzol, purified

RNA using RNeasy Mini Kit (Qiagen, Germantown, MD), reverse-transcribed it with qScript cDNA Synthesis Kit (QuantaBio, Beverly, MA), and performed PCR with GoTaq ${ }^{\circledR}$ qPCR Master Mix (Promega, Madison, WI). PCR primers are listed in Table S1. Gene expression levels were normalized to $18 \mathrm{~S}$ using the 2 -DDCt method ${ }^{44}$ and are presented as relative transcript levels.

\section{Immunohistochemistry and histomorphometry}

We anesthetized 8- to 12-week-old mice and harvested small and large intestine and rinsed them in PBS. Swiss rolls were prepared by rolling up the intestines around a pick stick from the pyloric sphincter toward cecum for small intestine, and from rectum to cecum for colon. Preparations were collected, fixed in 4\% paraformaldehyde for 2 -hr, dehydrated in 30\% sucrose overnight at $4^{\circ} \mathrm{C}$, embedded in OCT (Sakura, Torrance, CA), frozen to $-80^{\circ} \mathrm{C}$, and cut into $7-\mu \mathrm{m}$ sections. The primary and secondary antibodies used are listed in Table S2. FoxO1 staining was visualized by the Avidin/Biotin Blocking Kit (Vector Laboratories, CA) and biotinylated 
secondary antibody (BD, NJ) and fluorochrome-conjugated streptavidin (Vector Laboratories,

CA). Images were obtained with a confocal laser-scanning microscope (LSM 710, Carl Zeiss,

NY) using Zen software. Quantification was performed using ImageJ software [National

Institutes of Health]. The number of stained cells in the intestinal epithelium was counted

manually using the Adobe Photoshop 2021 software. The whole section of swiss roll was

scanned under 20x magnification using the Keyence BZ-X800 microscope. Images were stitched

using the Keyence Analyzer software. 200-300 villi-crypt unit or crypts were marked and

counted on the image of nuclei staining, followed by merging the image of insulin or C-peptide

staining and counting the positive signals only in the marked villi-crypt unit or crypts.

\section{Fluorescence-Activated Cell Sorting}

The tissue handling from murine intestinal epithelial cells isolation to fluorescence-

activated cell-sorted analysis has been described ${ }^{45}$ and was used in these experiments with slight

changes. A segment of the proximal intestine representing a $5-\mathrm{cm}$ region from 2 to $7 \mathrm{~cm}$ distal to

the pyloric sphincter was used for cell isolation. The intestinal segment was washed,

longitudinally cut open, and lightly rinsed to remove residual luminal contents. Tissue was

incubated in PBS containing $30 \mathrm{mmol} / 1 \mathrm{EDTA}$ and $1.5 \mathrm{mmol} / 1$ dithiothreitol on ice for $20 \mathrm{~min}$.

Intestinal tissue was transferred to a $15-\mathrm{ml}$ conical tube containing $5 \mathrm{ml}$ of $30 \mathrm{mmol} / \mathrm{l}$ EDTA

made in PBS, incubated at $37^{\circ} \mathrm{C}$ for $8 \mathrm{~min}$, then shaken by hand along the tube's long axis. After 
shaking, the remnant muscle layer was removed and the dissociated cells were pelleted at 1,000 g, resuspended in PBS supplemented with 10\% FBS, and centrifuged again. Cells were incubated in $10 \mathrm{ml}$ of modified HBSS containing $0.3 \mathrm{U} / \mathrm{ml}$ of dispase (Stem Cells Inc., CA) at $37^{\circ} \mathrm{C}$ for $10 \mathrm{~min}$ with intermittent shaking every $2 \mathrm{~min}$ for $15 \mathrm{~s}$ to dissociate epithelial sheets to single cells. After 10min, $1 \mathrm{ml}$ of FBS and 1,000 U DNase I (Sigma) were added to cell suspension, which was sequentially passed through $70-\mu \mathrm{m}$ then $40-\mu \mathrm{m}$ filters (Fisher Scientific, NH). Cells were pelleted, then resuspended in $10 \mathrm{ml}$ of HBSS to inactivate the dispase. Cells were centrifuged and resuspended at a concentration of $\sim 2 \times 10^{7}$ cells $/ \mathrm{ml}$ in FACS buffer [HBSS, EDTA (1mM), HEPES (25mM), 1\% BSA, Penicillin-Streptomycin]. SYTOX ${ }^{\mathrm{TM}}$ Red was used for the detection of dead cells (Invitrogen, MA). Cells were sorted using a BD Influx with a blue (488-nm) laser and 530/40 band pass filter, green (532-nm) laser and 580/30 band pass filter, red (640-nm) laser and 670/30 band pass filter to detect endogneous Green, Red proteins, and Sytox Red. RNA was isolated from 5,000 - 10,000 sorted cells, following to perform quantitative PCR analysis. To analyze the population of enteroendocrine cells, the isolated cells were first stained with live/dead cell staining kit (L34964), washed with 1x PBS and fixed in BD CytofixTM fixation buffer (BD Bioscience 554655) for 20 min. Cells were then permeabilized in permeabilization buffer (0.2\% saponin, $0.5 \% \mathrm{BSA}$ and $2 \mathrm{mM}$ EDTA in PBS) for $10 \mathrm{~min}$. The cells were incubated with the indicated primary antibodies diluted in the permeabilization buffer for $1 \mathrm{hr}$ at $4^{\circ} \mathrm{C}$, 
washed 3 times with permeabilization buffer, followed by the secondary antibodies diluted in permeabilization buffer for $30 \mathrm{~min}$ at $4{ }^{\circ} \mathrm{C}$. After 3 times of washes, $1 \times 10^{5}$ cells were analyzed using BD LSR FortessaTM Cell Analyzer.

\section{Gut Organoid cultures}

Mouse duodenum or ileum crypts were isolated. Duodenal samples included a 5 -cm segment of intestine 2 to $7 \mathrm{~cm}$ distal to the pyloric sphincter, ileum samples 2 to $7 \mathrm{~cm}$ proximal to the cecum, as described previously ${ }^{46}$. We seeded crypts into Matrigel (Corning, NY) to form mouse gut organoid (mGO) and cultured them for 48-hr prior to experiments. Basic medium was composed of Advanced DMEM/F12 containing 2 mM Glutamax, 10 mM HEPES, 100 U/ml penicillin/streptomycin, primocin $(100 \mu \mathrm{g} / \mathrm{ml})$ (Invivogen, CA). Complete growth medium was prepared by adding murine EGF (50ng/ml) (Gibco, TX), R-spondin-1 (conditioned medium 1m1/50ml) (gift by Akifumi Ootani, Stanford University, CA), Noggin (100ng/mL) (PeproTech, Inc, NJ), N-acetyl-L-cysteine (1mM) (Millipore Sigma, MA), B27 (1×)(Gibco, TX) to basic medium. The medium was replaced every 48-hr. All the experiments were done within 7 days after plating and done within 5 passages. For passage, every 7 days Matrigel was lysed with Cultrex ${ }^{\mathrm{TM}}$ Organoid Harvesting Solution (R\&D Systems $\left.{ }^{\mathrm{TM}}, \mathrm{MN}\right)$, and isolated $\mathrm{mGO}$, mechanically dissociated by pipetting, pelleted and re-plated in fresh Matrigel in 24-well plates at a 1:5 splitting ratio. Three hours treatment with DBZ $(5 \mu \mathrm{M})$ (Chiralix, Netherlands) was used 
to promote EEC differentiation, followed by replacing the medium with complete growth medium. Cpd10 $(1 \mu \mathrm{M})$ (Forkhead BioTherapeutics, NY) was used for single or dual treatment with DBZ, and Cpd10 was replaced every 48-hr.

\section{GLP-1 secretion}

Mouse ileum organoids were used for GLP-1 secretion assays. Hanks Buffered Salt

Solution supplemented with 10mM HEPES, $0.1 \%$ fatty acid-free bovine serum albumin was used as basic medium. mGO from 24-well plates were collected in $1.7 \mathrm{ml}$ tubes and incubated for 2-hr in basic medium, washed and incubated in $50 \mu$ l of basic medium with DPP4 inhibitor $(50 \mu \mathrm{M})$ for $1 \mathrm{~h}$. After collecting the supernatant, $10 \mathrm{mM}$ glucose solution in basic medium with DPP4 inhibitor was added to the tube, and incubated for $1 \mathrm{~h}$, after which we collected the supernatant. GLP-1 concentrations were determined by mouse GLP-1 ELISA Kit (Crystal Chem). mGOs were lysed in CelLytic ${ }^{\mathrm{TM}} \mathrm{M}$ (Sigma-Aldrich) for protein extraction, and protein content was normalized by total protein content quantified with BCA assay (Thermo scientific).

\section{Insulin secretion}

We collected mGO from 24-well plates, and placed them in ice-cold KRBH buffer (119 mM NaCl, 2.5 mM CaCl2, 115 1.19 mM KH2PO4, 1.19 mM Mg2SO4, 10 mM HEPES pH 7.4, $2 \% \mathrm{BSA}$, and 11.6 or $2.8 \mathrm{mM}$ glucose) and incubated at $37^{\circ} \mathrm{C}$ for $1-\mathrm{hr}$, followed by incubation in $2.8 \mathrm{mM}$ glucose for $1-\mathrm{hr}$ at $37^{\circ} \mathrm{C}$. After collecting the supernatant, $16.8 \mathrm{mM}$ glucose was added, 
and mGO incubated for 1-hr, followed by collection of the supernatant. Insulin was determined by mouse insulin ELISA (Mercodia). mGOs were lysed in CelLytic ${ }^{\mathrm{TM}} \mathrm{M}$ (Sigma-Aldrich) for protein extraction, and protein content normalized as above.

\section{Statistical Analysis}

Values are presented as means \pm standard deviation, and analyzed using Prism 8.2.1

(GraphPad Software, Inc.). We used unpaired Student's $t$-test for normally distributed variables

for comparisons between two groups, one-way ANOVA followed by Bonferroni post-hoc test

for multiple comparisons, and Pearson's correlation coefficient to investigate the relationship

between two variables. We used a threshold of $p_{\text {alpha (2-tailed })}<0.05$ to declare statistical

significance. 
Duality of interest. D.A. is founder, director, chair of the advisory board, and holds an equity interest in Forkhead Biotherapeutics, Inc. Y.-K.L. and S.B. are employees of Forkhead Biotherapeutics, Inc. Hua V. Lin is a former employee of Forkhead Biotherapeutics. T.K., D.A., H.V.L., Y.-K.L., and S.B. are inventors on a patent application describing some the findings. Funding: Supported by a grant from the JPB Foundation and a sponsored research agreement with Forkhead BioTherapeutics Inc. W.M.M. is supported by 1K01DK121873 from the NIDDK.

Author Contribution: T.K. performed experiments, analyzed results, wrote and edited the manuscript; Y.K.L, N.S, and B.D performed in vivo experiments, analyzed results, and wrote the manuscript; W.M.M. and W.D. performed experiments, H.L. and H.W. edited the manuscript, R.L and S.B. oversaw research related to Cpd10 experiments, D.A. oversaw research and wrote the manuscript.

Acknowledgments: We thank members of the Accili laboratory, Dr. Utpal B. Pajvani for insightful discussions of the data, and Mr. Thomas Kolar and Ms. Ana Maria Flete (Columbia University), and Carmen Lam, Kasia Dover, Xiaoming Xu for exceptional technical support. 


\section{References}

1. Krentz, N.A.J., Shea, L.D., Huising, M.O. \& Shaw, J.A.M. Restoring normal islet mass and function in type 1 diabetes through regenerative medicine and tissue engineering. The Lancet Diabetes \& Endocrinology 9, 708-724 (2021).

2. Helman, A. \& Melton, D.A. A Stem Cell Approach to Cure Type 1 Diabetes. Cold Spring Harb Perspect Biol (2020).

3. McKimpson, W.M. \& Accili, D. Reprogramming Cells to Make Insulin. J Endocr Soc 3, 12141226 (2019).

4. Nair, G.G., Tzanakakis, E.S. \& Hebrok, M. Emerging routes to the generation of functional $\beta$ cells for diabetes mellitus cell therapy. Nature Reviews Endocrinology (2020).

5. Chen, Y.J., et al. De novo formation of insulin-producing "neo-beta cell islets" from intestinal crypts. Cell Rep 6, 1046-1058 (2014).

6. Talchai, C., Xuan, S., Kitamura, T., DePinho, R.A. \& Accili, D. Generation of functional insulinproducing cells in the gut by Foxo1 ablation. Nat Genet 44, 406-412, S401 (2012).

7. Bouchi, R., et al. FOXO1 inhibition yields functional insulin-producing cells in human gut organoid cultures. Nature communications 5, 4242 (2014). 
8. Cheung, A.T., et al. Glucose-dependent insulin release from genetically engineered K cells.

Science 290, 1959-1962 (2000).

9. Iweala, O.I. \& Nagler, C.R. Immune privilege in the gut: the establishment and maintenance of non-responsiveness to dietary antigens and commensal flora. Immunol Rev 213, 82-100 (2006).

10. Huang, H. \& Tindall, D.J. Dynamic FoxO transcription factors. J Cell Sci 120, 2479-2487 (2007).

11. Brent, M.M., Anand, R. \& Marmorstein, R. Structural basis for DNA recognition by FoxO1 and its regulation by posttranslational modification. Structure 16, 1407-1416 (2008).

12. Li, J., et al. Mechanism of forkhead transcription factors binding to a novel palindromic DNA site. Nucleic Acids Res 49, 3573-3583 (2021).

13. Lee, Y.K., et al. FOXO1 inhibition synergizes with FGF21 to normalize glucose control in diabetic mice. Molecular metabolism 49, 101187 (2021).

14. Langlet, F., et al. Selective Inhibition of FOXO1 Activator/Repressor Balance Modulates Hepatic Glucose Handling. Cell 171, 824-835 e818 (2017).

15. Kitamura, T., et al. A Foxo/Notch pathway controls myogenic differentiation and fiber type specification. J Clin Invest 117, 2477-2485 (2007).

16. Pajvani, U.B., et al. Inhibition of Notch uncouples Akt activation from hepatic lipid accumulation by decreasing mTorc1 stability. Nat Med 19, 1054-1060 (2013). 
17. Murtaugh, L.C., Stanger, B.Z., Kwan, K.M. \& Melton, D.A. Notch signaling controls multiple steps of pancreatic differentiation. Proc Natl Acad Sci U S A 100, 14920-14925 (2003).

18. Petersen, N., et al. Targeting development of incretin-producing cells increases insulin secretion. Journal of Clinical Investigation 125, 379-385 (2015).

19. Pajvani, U.B., et al. Inhibition of Notch signaling ameliorates insulin resistance in a FoxO1dependent manner. Nat Med 17, 961-967 (2011).

20. Schonhoff, S.E., Giel-Moloney, M. \& Leiter, A.B. Neurogenin 3-expressing progenitor cells in the gastrointestinal tract differentiate into both endocrine and non-endocrine cell types. Dev Biol 270, 443-454 (2004).

21. Madison, B.B., et al. Cis elements of the villin gene control expression in restricted domains of the vertical (crypt) and horizontal (duodenum, cecum) axes of the intestine. J Biol Chem 277, $33275-33283$ (2002).

22. Yoshioka, M., Kayo, T., Ikeda, T. \& Koizumi, A. A novel locus, Mody4, distal to D7Mit189 on chromosome 7 determines early-onset NIDDM in nonobese C57BL/6 (Akita) mutant mice.

Diabetes 46, 887-894 (1997).

23. Petersen, N., et al. Targeting development of incretin-producing cells increases insulin secretion. J Clin Invest 125, 379-385 (2015). 
24. Wu, C.X., et al. Notch Inhibitor PF-03084014 Inhibits Hepatocellular Carcinoma Growth and Metastasis via Suppression of Cancer Stemness due to Reduced Activation of Notch1-Stat3.

Molecular Cancer Therapeutics 16, 1531-1543 (2017).

25. Accili, D. Insulin Action Research and the Future of Diabetes Treatment: The 2017 Banting

Medal for Scientific Achievement Lecture. Diabetes 67, 1701-1709 (2018).

26. Kitamura, Y.I., et al. FoxO1 protects against pancreatic beta cell failure through NeuroD and MafA induction. Cell Metab 2, 153-163 (2005).

27. Kitamura, T., et al. Regulation of pancreatic juxtaductal endocrine cell formation by FoxO1. Mol Cell Biol 29, 4417-4430 (2009).

28. Al-Masri, M., et al. Effect of forkhead box O1 (FOXO1) on beta cell development in the human fetal pancreas. Diabetologia 53, 699-711 (2010).

29. Zhao, M., et al. Protein O-GlcNAc Modification Links Dietary and Gut Microbial Cues to the Differentiation of Enteroendocrine L Cells. Cell Reports 32, 108013 (2020).

30. Chen, Y.J., et al. De Novo Formation of Insulin-Producing "Neo-beta Cell Islets" from Intestinal Crypts. Cell reports 6, 1046-1058 (2014).

31. Ariyachet, C., et al. Reprogrammed Stomach Tissue as a Renewable Source of Functional beta Cells for Blood Glucose Regulation. Cell stem cell 18, 410-421 (2016). 
32. van Es, J.H., et al. Notch/gamma-secretase inhibition turns proliferative cells in intestinal crypts

and adenomas into goblet cells. Nature 435, 959-963 (2005).

33. Pajvani, U.B., et al. Inhibition of Notch signaling ameliorates insulin resistance in a FoxO1-

dependent manner. Nature Medicine 17, 961-967 (2011).

34. Calissi, G., Lam, E.W. \& Link, W. Therapeutic strategies targeting FOXO transcription factors.

Nat Rev Drug Discov 20, 21-38 (2021).

35. Paik, J.H., et al. FoxOs are lineage-restricted redundant tumor suppressors and regulate

endothelial cell homeostasis. Cell 128, 309-323 (2007).

36. Kerdiles, Y.M., et al. Foxo transcription factors control regulatory T cell development and

function. Immunity 33, 890-904 (2010).

37. Trinh, D.L., et al. Analysis of FOXO1 mutations in diffuse large B-cell lymphoma. Blood 121,

3666-3674 (2013).

38. Seda, V., et al. FoxO1-GAB1 axis regulates homing capacity and tonic AKT activity in chronic

lymphocytic leukemia. Blood 138, 758-772 (2021).

39. Zhou, W., et al. FoxO4 inhibits NF-kappaB and protects mice against colonic injury and

inflammation. Gastroenterology 137, 1403-1414 (2009).

40. Malik, S., et al. Transcription factor Foxo1 is essential for IL-9 induction in T helper cells.

Nature communications $\mathbf{8 ,} 815$ (2017). 
41. Teoh, S.L. \& Das, S. Notch Signalling Pathways and Their Importance in the Treatment of

Cancers. Curr Drug Targets 19, 128-143 (2018).

42. Accili, D. Whither Type 1 Diabetes? N Engl J Med 383, 2078-2079 (2020).

43. Muzumdar, M.D., Tasic, B., Miyamichi, K., Li, L. \& Luo, L. A global double-fluorescent Cre reporter mouse. Genesis 45, 593-605 (2007).

44. Livak, K.J. \& Schmittgen, T.D. Analysis of relative gene expression data using real-time quantitative PCR and the 2(-Delta Delta C(T)) Method. Methods 25, 402-408 (2001).

45. Magness, S.T., et al. A multicenter study to standardize reporting and analyses of fluorescenceactivated cell-sorted murine intestinal epithelial cells. Am J Physiol Gastrointest Liver Physiol 305, G542-551 (2013).

46. Sato, T. \& Clevers, H. Primary Mouse Small Intestinal Epithelial Cell Cultures. in Epithelial Cell Culture Protocols: Second Edition (eds. Randell, S.H. \& Fulcher, M.L.) 319-328 (Humana Press, Totowa, NJ, 2013). 
FIGURE LEGENDS

Figure 1. Gut FoxO1 ablation expands the EEC progenitor pool

(A, B) Representative images of GFP and RFP immunofluorescence in intestinal duodenum sections of TG-WT (A) and TG-NFKO (B) mice. (C) Representative FACS plot of TG-WT gut epithelial cells. P4: $\mathrm{RFP}^{+}$; P5: RFP ${ }^{+} \mathrm{GFP}^{+}$; P6: GFP ${ }^{+}$. (D-F) qPCR of ChgA (D), Tph1 (E), and FoxO1 (F) using mRNA from sorted P4, P5, and P6 cells. N=3 for each group. (G-J) Time course of induction of P5 and P6 population after DBZ treatment. White and red circles indicate TG-WT and TG-NFKO, respectively. $3 \times 10^{5}$ cells were analyzed in each experiment, and each group consists of biological triplicates. Merged FACS plots of three samples from TG-WT (I) and TG-NFKO (J) are shown. $(\mathrm{K}, \mathrm{L})$ Representative GFP and RFP immunofluorescence images in intestinal duodenum sections of TG-WT (K) and TG-NFKO (L) after 96hr DBZ treatment. TG-WT: ACTB-tdTomato,-EGFP; Neurog3-Cre: TG-NFKO, ACTB-tdTomato-EGFP; Neurog3-Cre $(+):$ FoxO1 $^{\mathrm{fl} / \mathrm{fl}}$. Data are presented as means \pm SD. Scale bar $=50 \mu \mathrm{m} . *=\mathrm{p}<0.05 ; * *=\mathrm{p}<$ $0.01 ; * * *=\mathrm{p}<0.001$

Figure 2. Time course of EEC differentiation after FoxO1 ablation (A-D) Time course analysis of FoxO1 (A), Ngn3 (B), ChgA (C), and Tph1 (D) expression in mouse organoids after DBZ treatment. All the data were normalized by $18 \mathrm{~S}$. White, blue and red filled circles indicate FoxO1 ${ }^{\mathrm{fl} / \mathrm{fl}}$, NFKO, VFKO mice $\mathrm{mGO}$, respectively. (E-G) Immunohistochemistry of FoxO1 and 5HT (E), GLP-1 and 5HT (F), and C-peptide (G) in mGO from $F_{0 x O} \mathrm{fl}^{1 / f 1}$, or VFKO mice after DBZ treatment. NFKO, Neurog3-Cre $(+)$; FoxO1 1 fl/fl: VFKO, Villin-Cre $(+) ;$ FoxO1 $^{\mathrm{fl} / \mathrm{fl}}$. Scale bar $=50 \mu \mathrm{m}$. DAPI counterstains nuclei. Data are presented as means \pm SD. $\quad *=\mathrm{p}<0.05 ; * *=\mathrm{p}<0.01 ; * * *=\mathrm{p}<0.001 ; * * * *=\mathrm{p}<0.0001$

Figure 3. Metabolic effects of gut FoxO1 ablation 
(A-B) Immunohistochemistry with GLP-1 (A), GIP (B), and C-peptide antibodies (C) in ileum (A), or duodenum (B and C) of FoxO $\mathrm{fl}^{\mathrm{fl} f \mathrm{l}}$ or VFKO mice. (D-G) Oral (D) or intra-peritoneal (E) glucose tolerance tests ( $\mathrm{g} / \mathrm{kg}$ glucose infusion) after a 4-hr fast and area under curve measurements of (F-G). (H) Plasma insulin levels during oral glucose tolerance tests are shown. White and red circles represent FoxO ${ }^{\mathrm{fl} / \mathrm{fl}}$ and VFKO mice, respectively. VFKO, Vil-Cre (+); FoxO $1^{\mathrm{fl} / \mathrm{fl}}$ : Scale bar $=50 \mu \mathrm{m}$. DAPI counterstains nuclei. Data are presented as means $\pm \mathrm{SD} *=\mathrm{p}$ $<0.05 ; * * *=\mathrm{p}<0.001$

Figure 4. Gut FoxO1 ablation reverses hyperglycemia in INS2Akita/+ (A) Blood glucose levels in INS2 $2^{\text {Akita/+ }}$ mice with or without FoxO1 $1^{\mathrm{fl} / f 1}$ and VFKO alleles $(\mathrm{n}=14$ - 20 in each group). (B) Blood glucose levels in INS2 $2^{\text {Akita/+ }}$ and INS2 $2^{\text {Akita/+}}$ VFKO mice at $16-24$ weeks ages after 4-hr fast, followed by 1 -hr refeeding $(n=5$ in each group). (C) Plasma insulin levels in INS2 $2^{\text {Akita/+ }}$ and INS2 $2^{\text {kkita/+ }}$ VFKO mice at 8,10 , and 12 weeks ages ( $\mathrm{n}=6$ in each group). (D-E) Oral glucose (1 g/kg glucose infusion) tolerance tests (OGTT) after 16-hr fast of INS2 ${ }^{\text {Akita/+ }}$ (white circles) and INS2 ${ }^{A k i t a /+}$ VFKO mice (red circles) following administration of vehicle (D) or GLP-1 antagonist, exendin-9 by i.p. (E) ( $n=6$ and 7 in each group). (F) Area under the curve (AUC) measurements of OGTT in (D) and (E). (G-I) Immunohistochemistry of GLP-1 (G), GIP (H), and C-peptide (I) in ileum $(\mathrm{G})$, or duodenum $(\mathrm{H}-\mathrm{I})$ of $I N S 2^{\text {Akita/+ }}$ and $I N S 2^{\text {Akita/+}}$ VFKO mice. VFKO, Vil-Cre (+); FoxO1 $1^{\mathrm{fl} / \mathrm{fl}}$ : Akita-WT, INS2 ${ }^{\text {Akita/+}}$; FoxO1 ${ }^{\mathrm{fl} / \mathrm{fl}}$ : Akita-VFKO, INS2 ${ }^{\text {Akita/++ }}$; VilCre (+): FoxO1 $1^{\mathrm{fl} / \mathrm{fl}}$. Data are presented as means $\pm \mathrm{SD}$. Scale bar $=50 \mu \mathrm{m}$, DAPI counterstains nuclei. $*=\mathrm{p}<0.05 ; * *=\mathrm{p}<0.01 ; * * *=\mathrm{p}<0.001$ by two-factor ANOVA. 
Figure 5. $\beta$-like cell conversion by dual FoxO1-Notch inhibition

(A-D) Time course of mGO gene expression following treatment with vehicle (white circles) or Cpd10 (blue circles). (A), Ngn3 (B), ChgA (C), and Tph1 mRNA (D) after DBZ treatment. (EF) GLP-1 secretion from FoxO1 $1^{\mathrm{fl} / \mathrm{fl}}$ or VFKO mice $\mathrm{mGO}$ treated with vehicle (white and blue bars) or DBZ (black and red bars) for 96-hr (E). GLP-1 secretion from WT mGO with or without Cpd10 and/or DBZ treatment for $96 \mathrm{hr}(\mathrm{F}) .(\mathrm{G}-\mathrm{H})$ Insulin secretion from NFKO or VFKO mGO treated with vehicle (white squares or triangles) or DBZ (red squares or triangles) for 96-hr (G). Insulin secretion from WT mGO with or without Cpd10 and/or DBZ treatment for 96-hr (H). Data are presented as means $\pm \mathrm{SD} . *=\mathrm{p}<0.05 ; * * *=\mathrm{p}<0.001$

Figure 6. Pharmacological inhibition of Foxol and Notch in $A K I T A^{\text {Ins2/+ }}$ mice 8- to 9-week-old Akita mice were randomized into 4 groups based on glucose levels and body weight. Mice were treated with vehicle, Cpd10 (50 mg/kg/), or PF-03084014 (150 mg/kg) or Cpd10 (50mg/kg) + PF-03084014 (150mg/kg) for 5 days, twice daily. (A) Body weight measured before morning dosing. (B) Ad libitum glucose levels measured between 4 and 5 pm on day 1 and day 4. N=6-13 per group. Data was combined from 4 experiments conducted under the same conditions and presented as means \pm SEM. $* *=\mathrm{p}<0.01 ; * * *=\mathrm{p}<0.001 ; * * * *=\mathrm{p}<$ 0.0001 by two-way ANOVA. NS: not significant. (C) Oral glucose tolerance (OGTT) test. $2 \mathrm{~g} / \mathrm{kg}$ of glucose solution was administered orally after a 4-hr fast (8:30am to 12:30 pm), and glucose levels were measured at $0,15,30,60$, and $120 \mathrm{~min}$. (D) Total AUC. $* *=p<0.01$ by one-way ANOVA. NS: not significant. (E) Blood was collected at 0 and 30 min during OGTT from tail clip capillary and plasma insulin level analyzed by ELISA. $* * *=\mathrm{p}<0.001$ by t-test. NS: not significant. (F-M) Representative intestinal immunohistochemistry after 10-day treatment. (F, G) 
Insulin (red) and 5-HT (green), (H, I) PC2 (red) and 5-HT (green), (J, K) C-peptide and (L, M)

GLP-1 (red) and 5-HT (green) staining of vehicle group (left panel, F, H, J, and L) and

Cpd10/PF-03084014-treated group (right panel, G, I, K, M). Scale bar $=50 \mu \mathrm{m}(\mathrm{N})$ Gene

expression in isolated epithelial cells for the markers indicated. (O-P) $\operatorname{Insulin}^{+}(\mathrm{O})$ and Cpeptide $^{+}$cells $(\mathrm{P})$ quantification in villi and crypts. 
Figure. 1

A

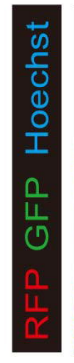

B

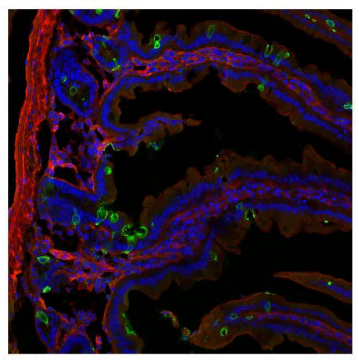

K

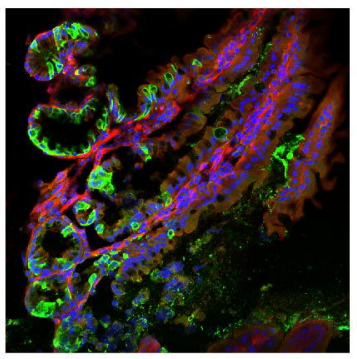

L

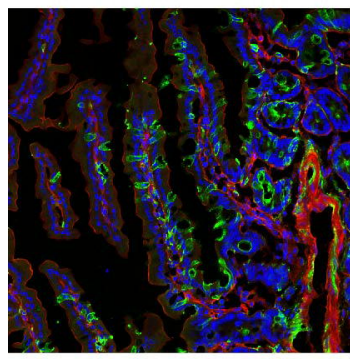

E

D

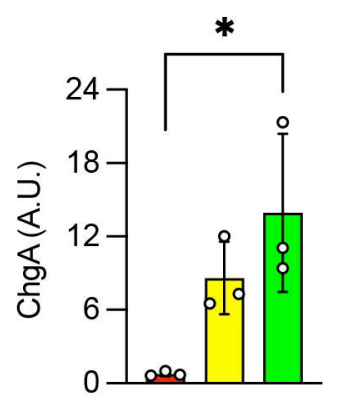

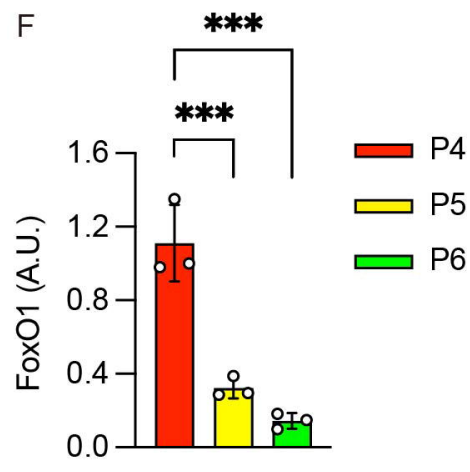

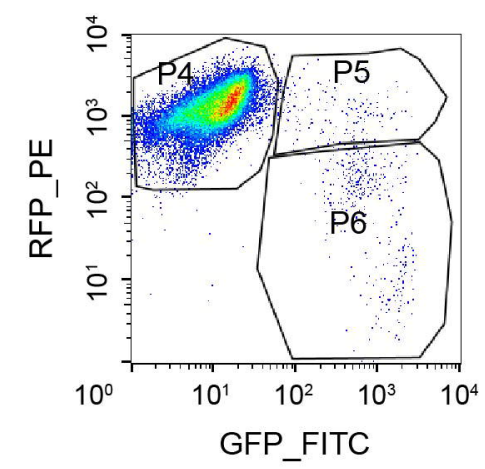

G

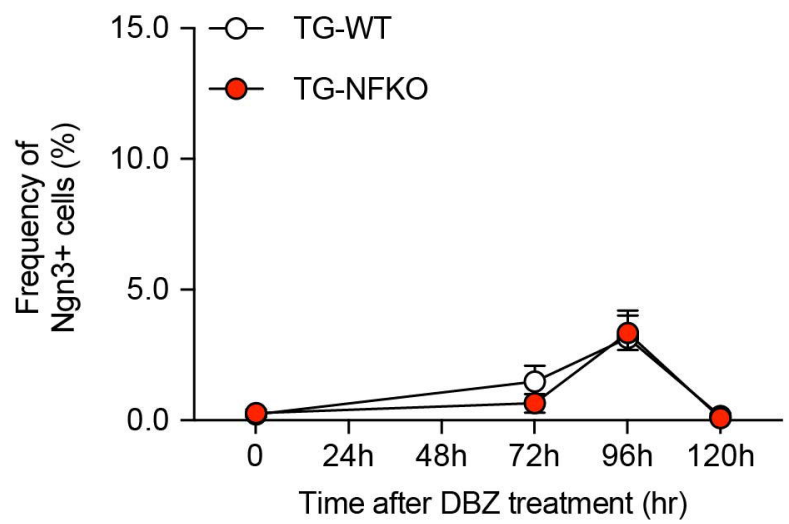

$\mathrm{H}$

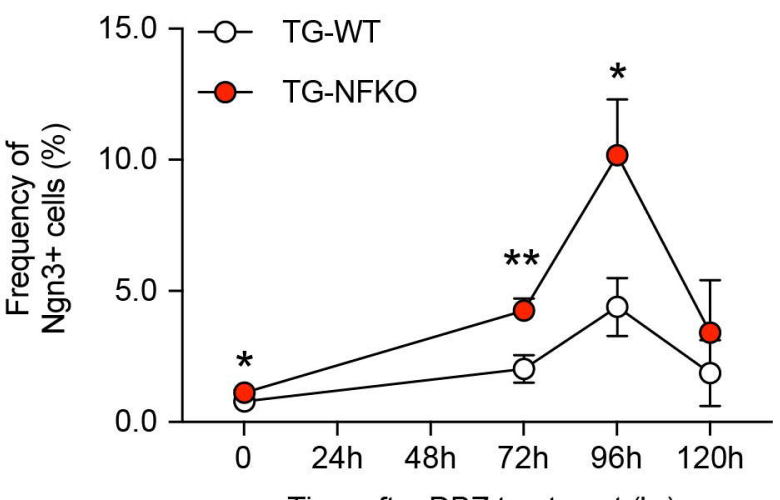

Time after DBZ treatment (hr)

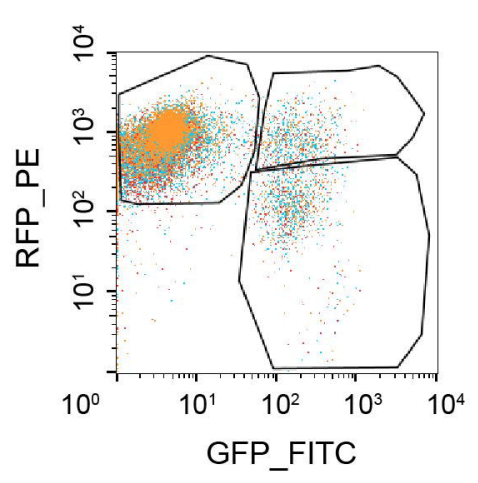

J

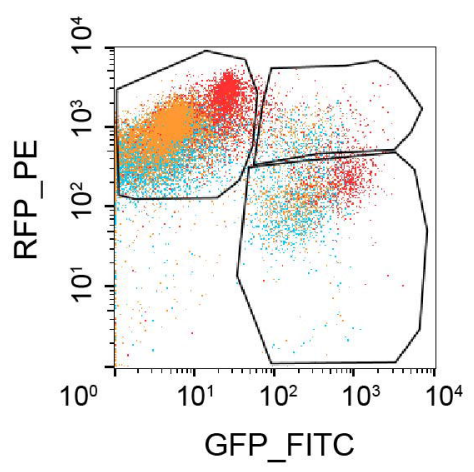


Figure. 2

A

B

C

D

$-0-F o x O 1^{\text {fl/fl }}$

$\multimap-V F K O \rightarrow-N F K O$
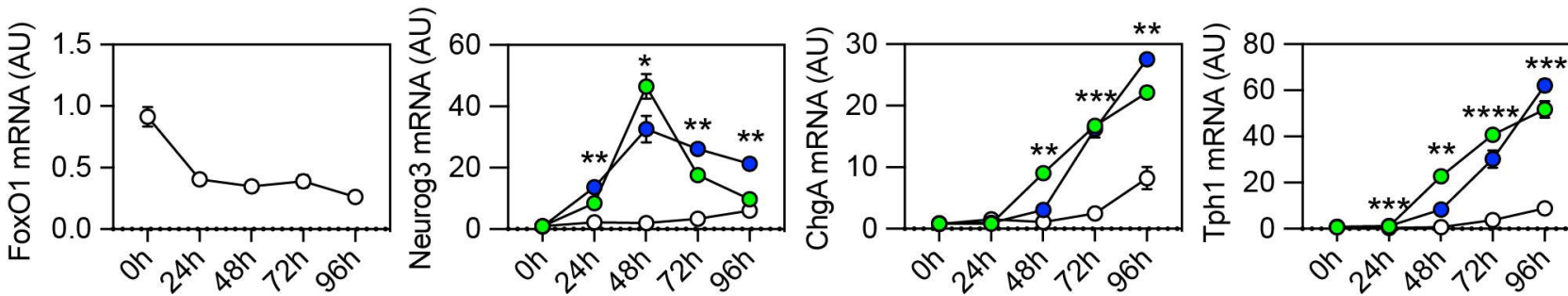

Time after DBZ treatment (hr)

E
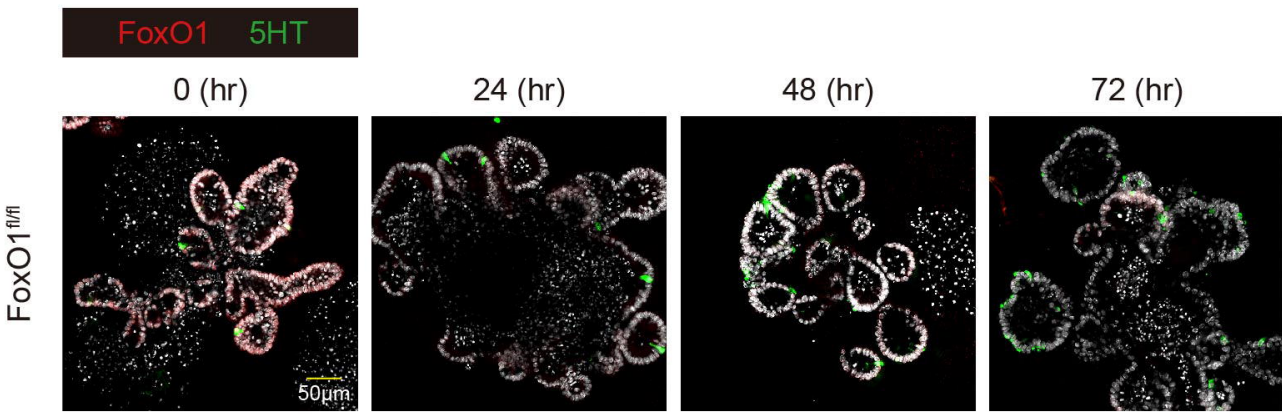

$96(\mathrm{hr})$
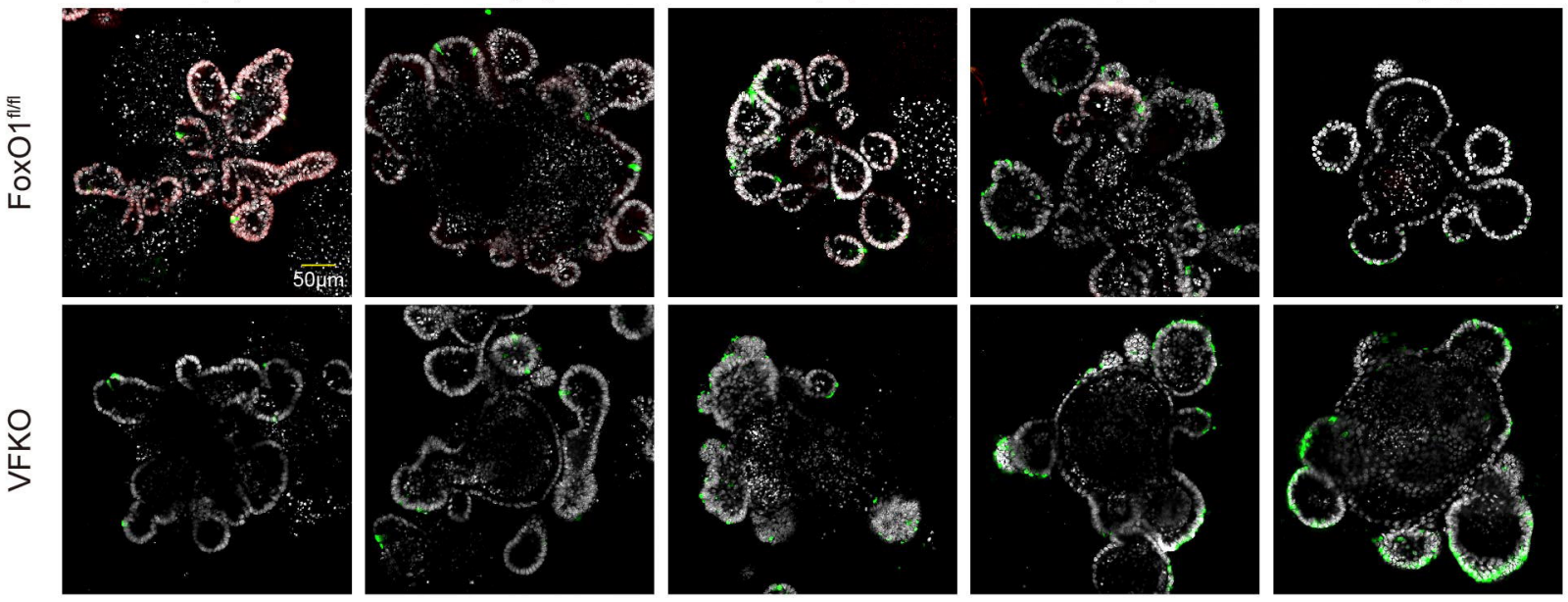

F

G

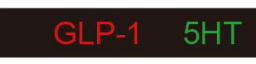

0 (hr)
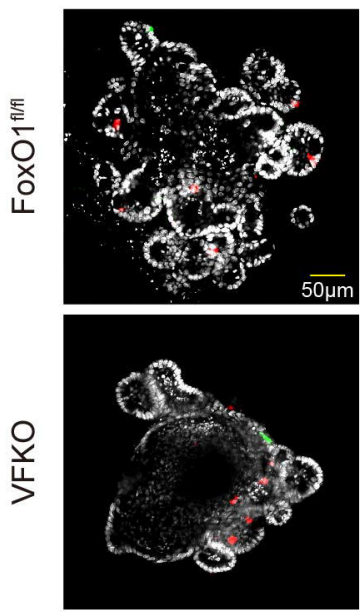

96 (hr)
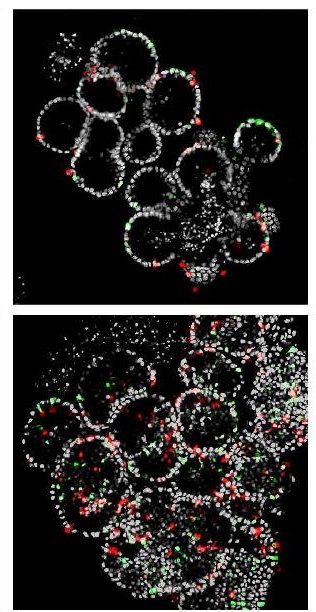
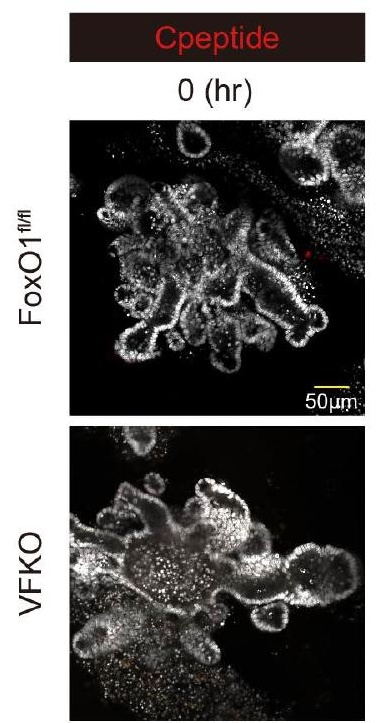

96 (hr)
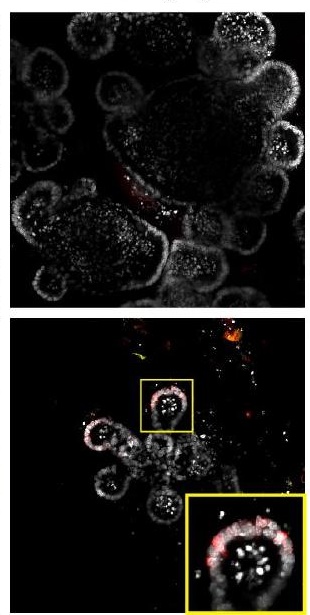
Figure. 3

A
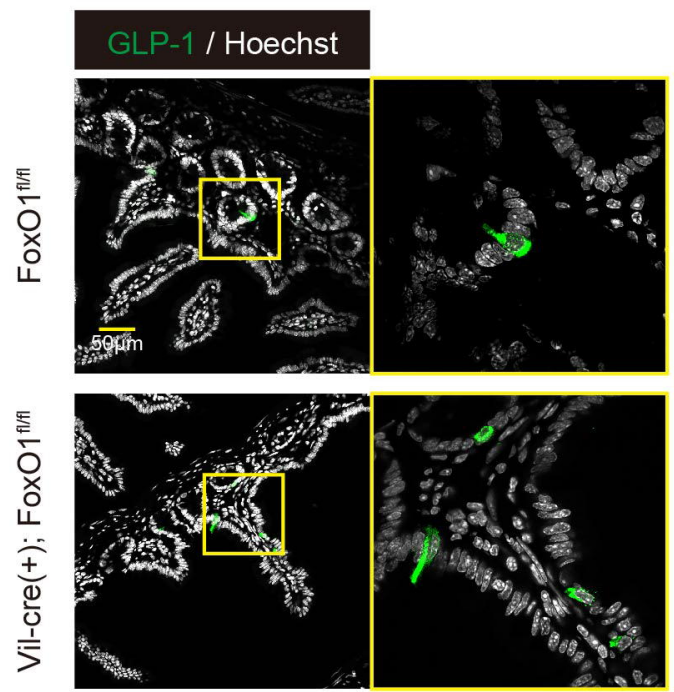

D

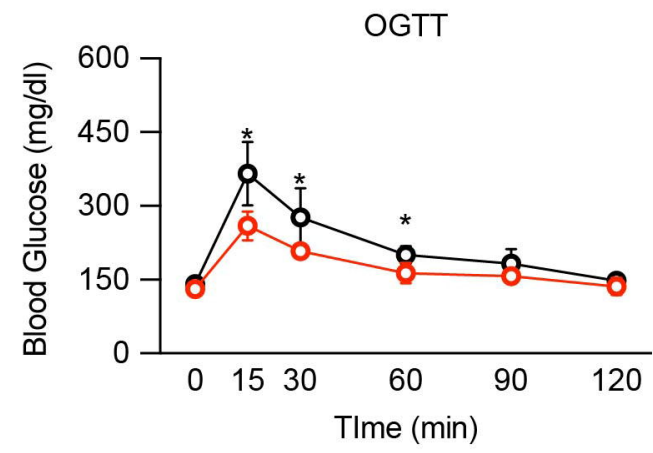

F
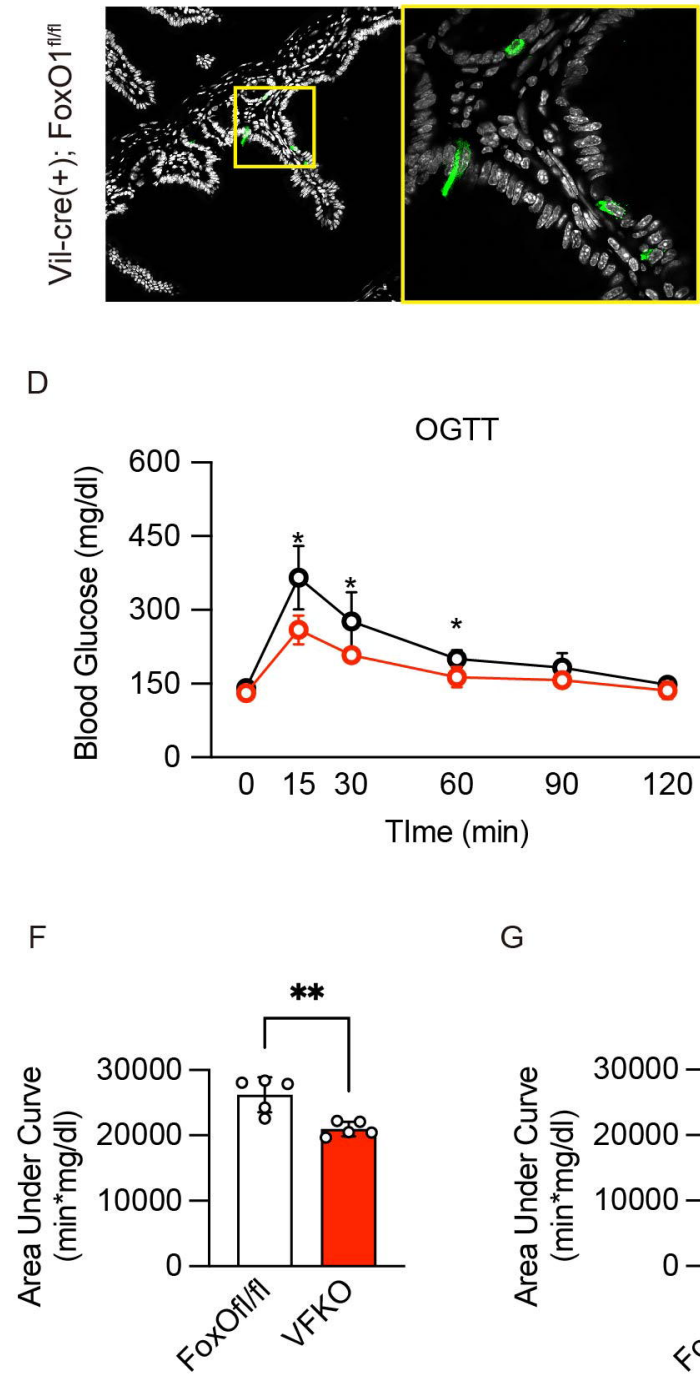

B
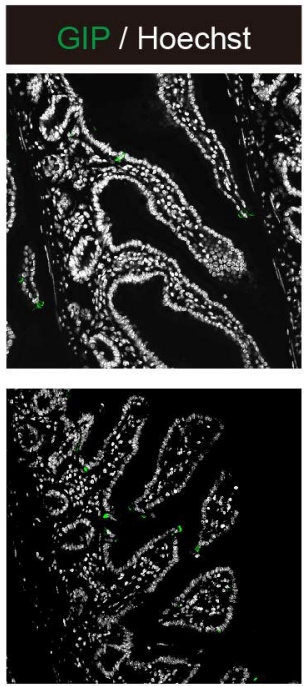

E

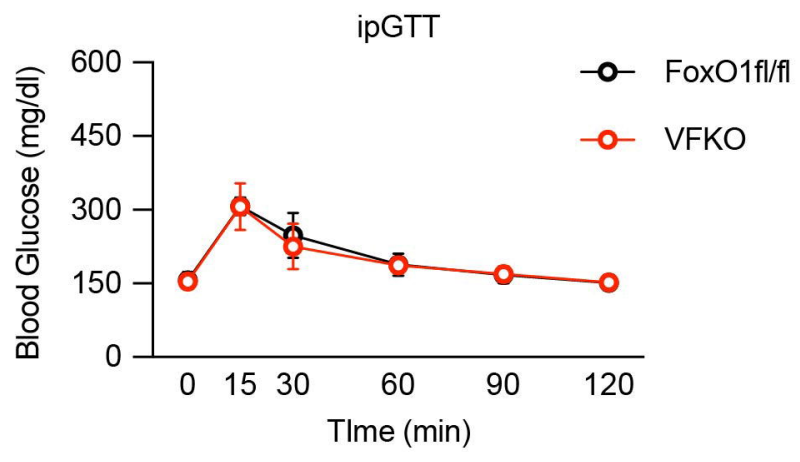

$\mathrm{H}$

C
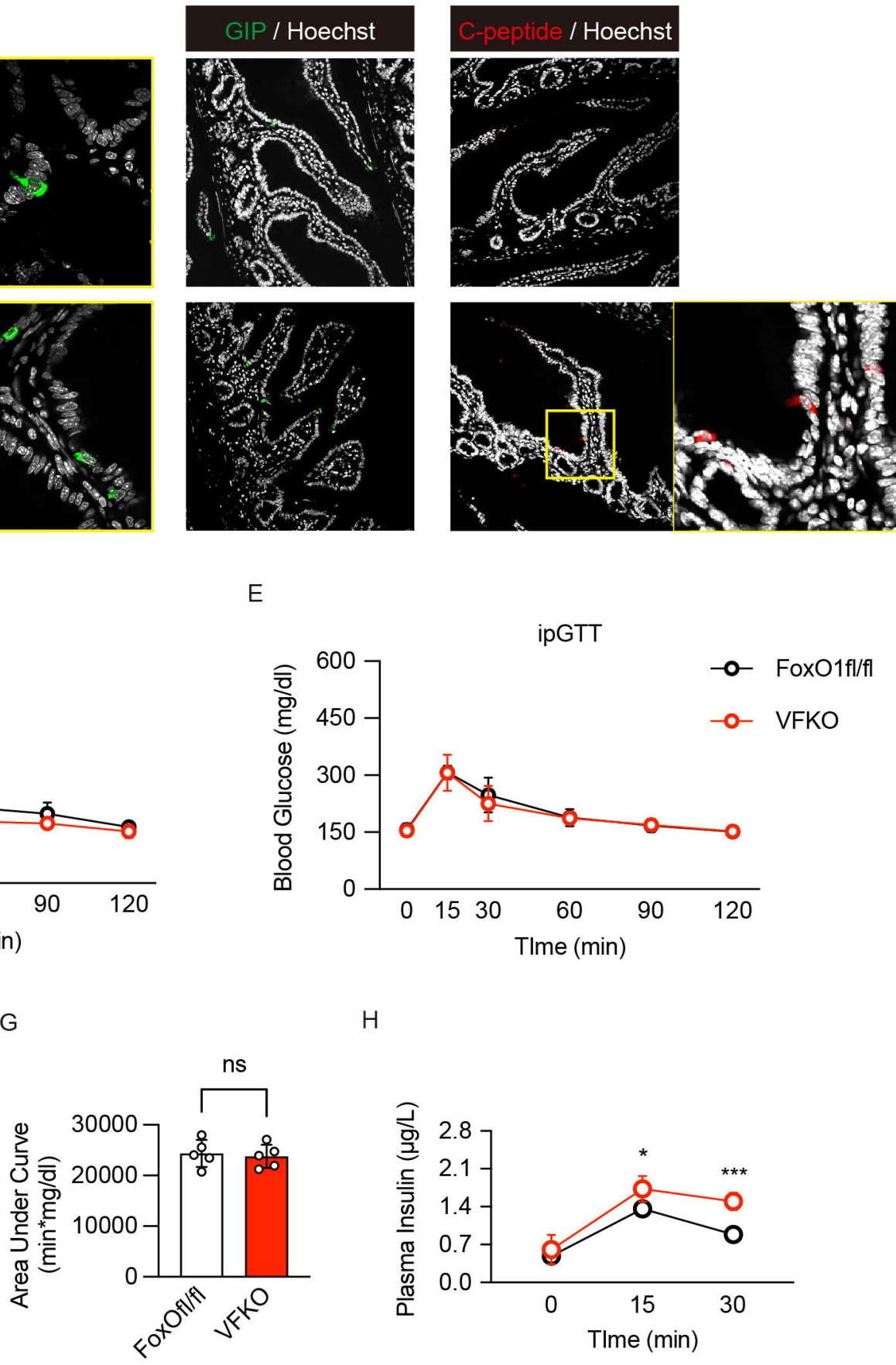
A

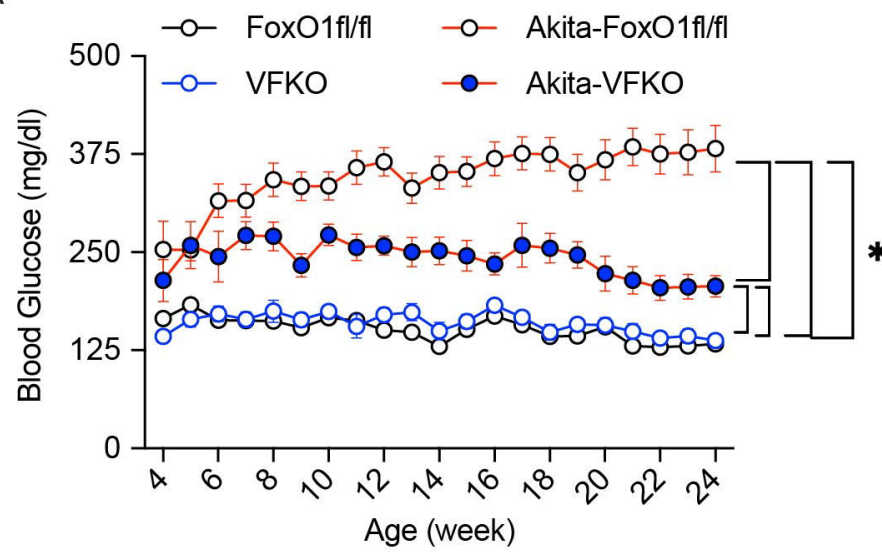

B

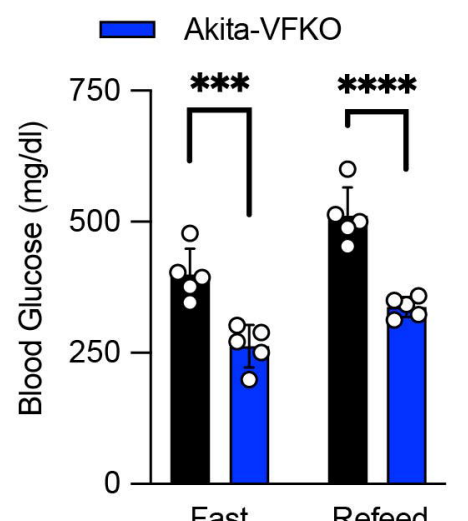

G

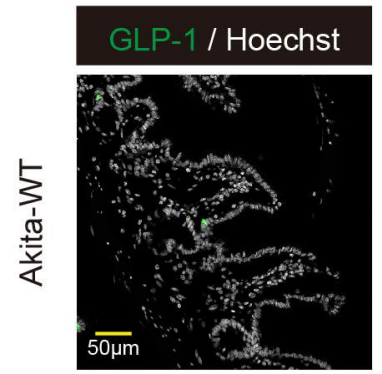

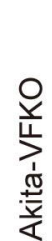

C

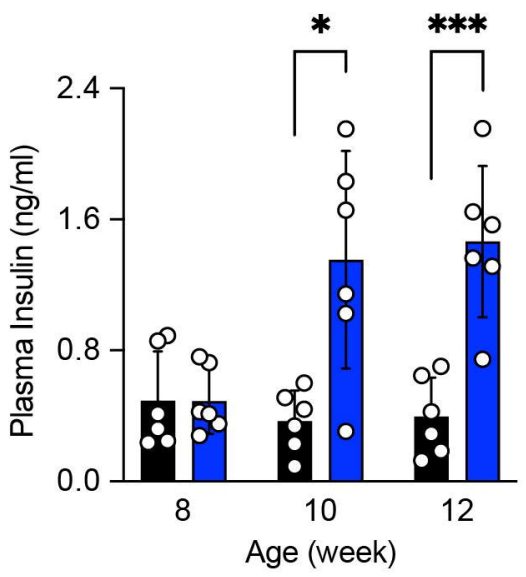

Age (week)

$\mathrm{H}$
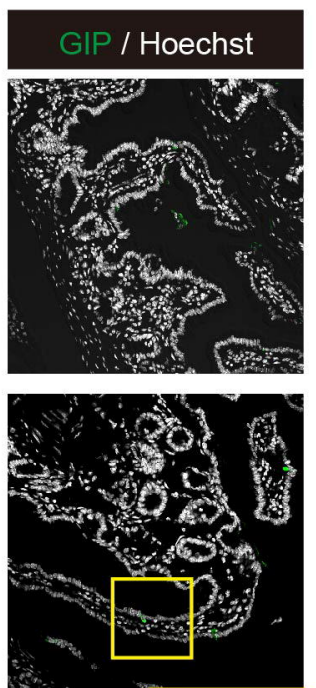

$* * * *$

E

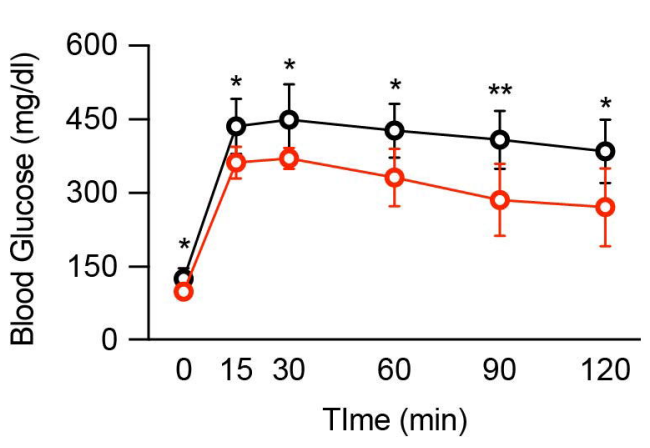

-o- Akita: FoxO1fl/fl

-o- Akita: VFKO

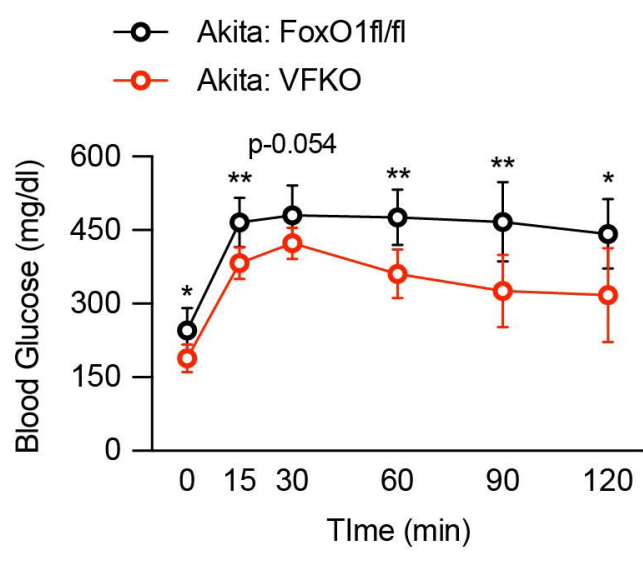

F

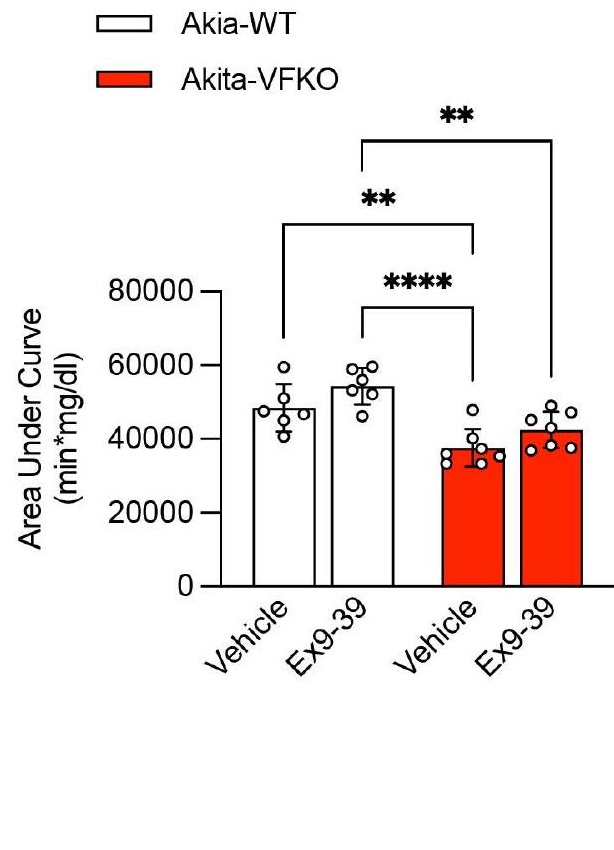


Figure. 5
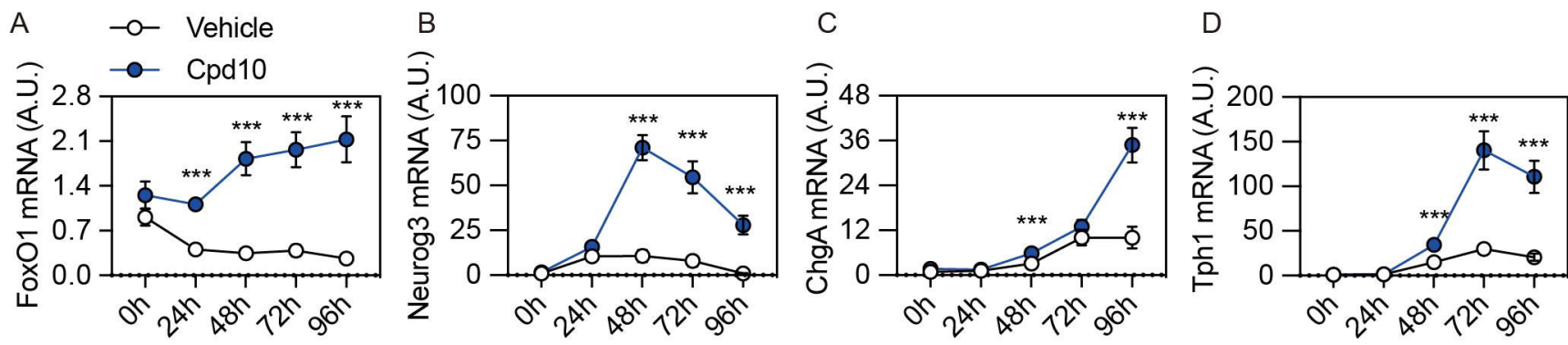

Time after DBZ treatment (hr)

E

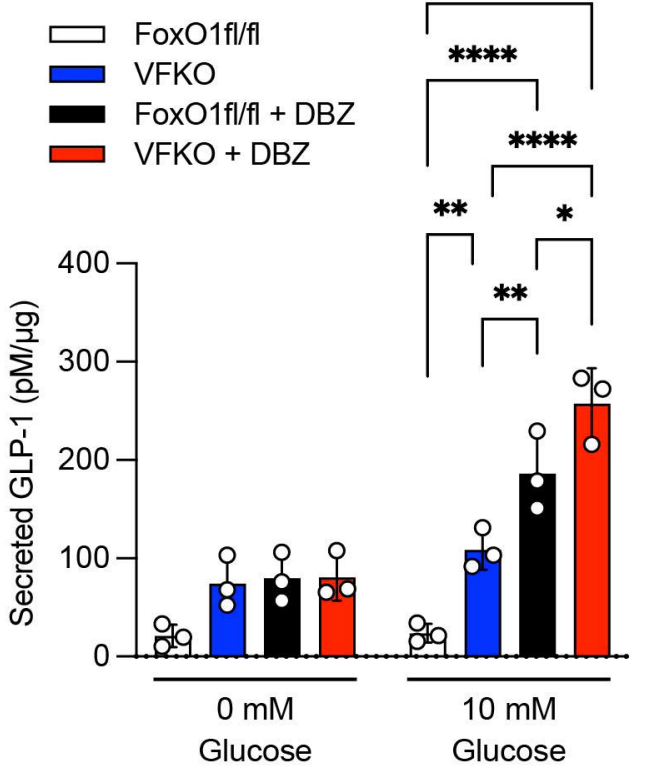

G

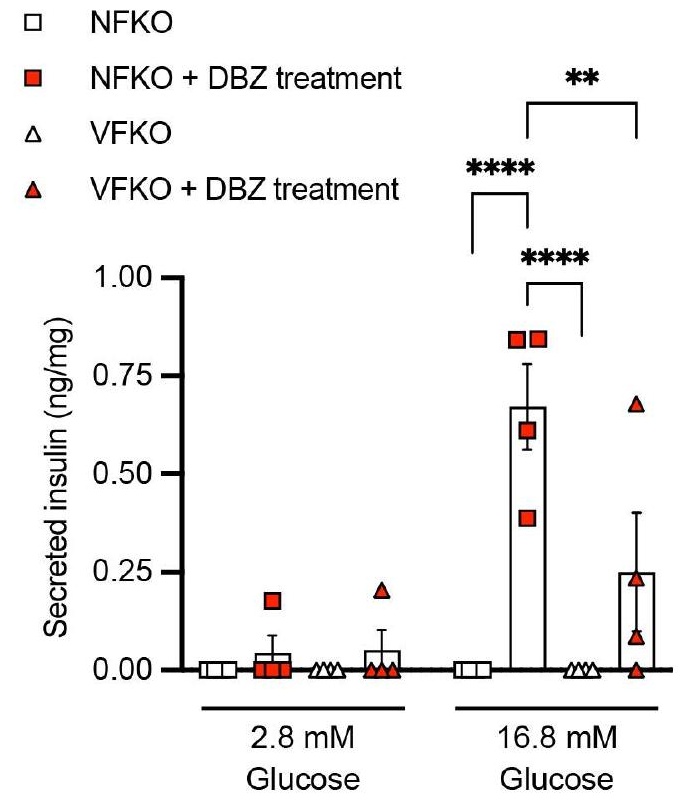

H
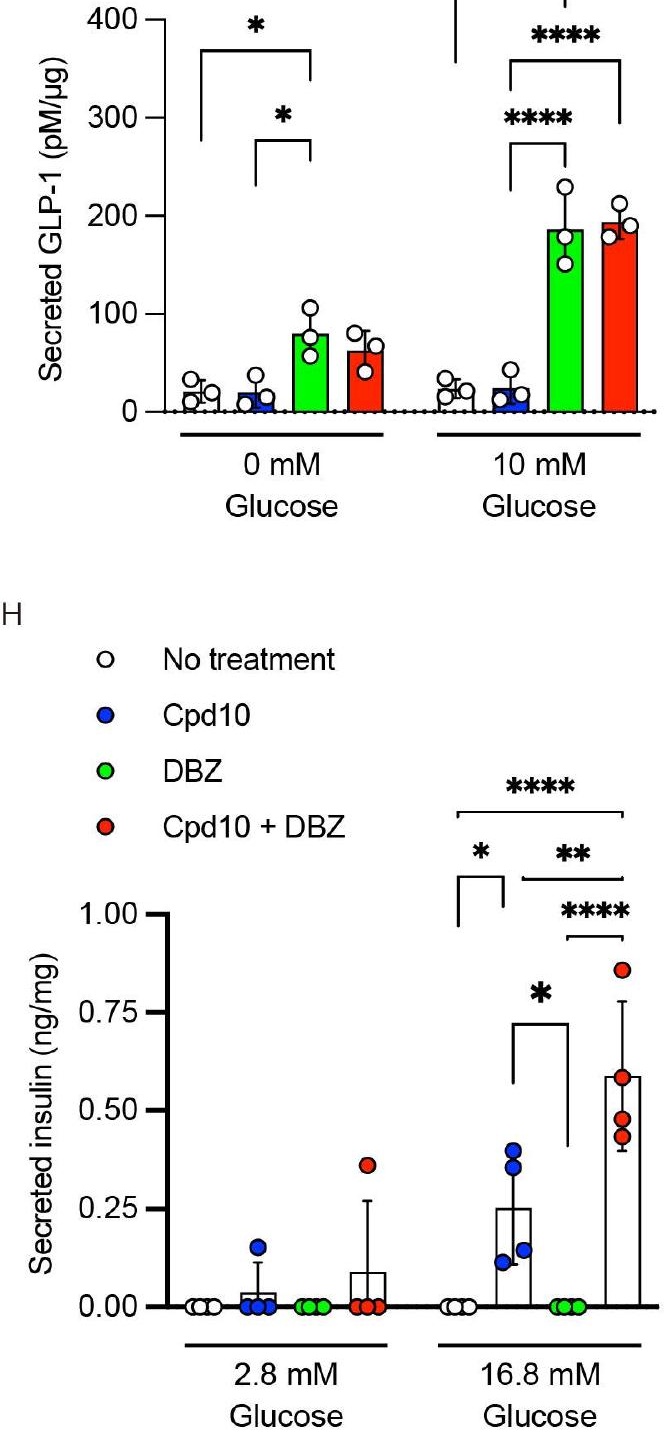
A

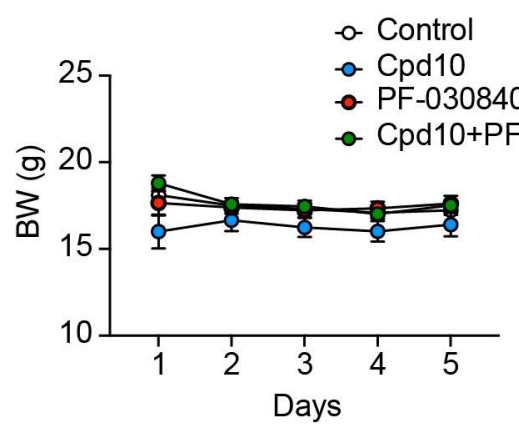

C

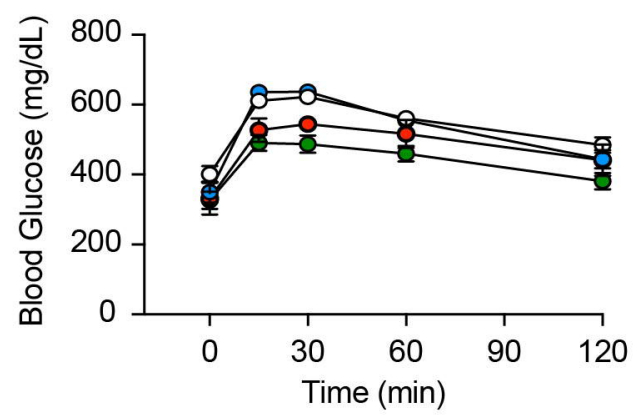

F
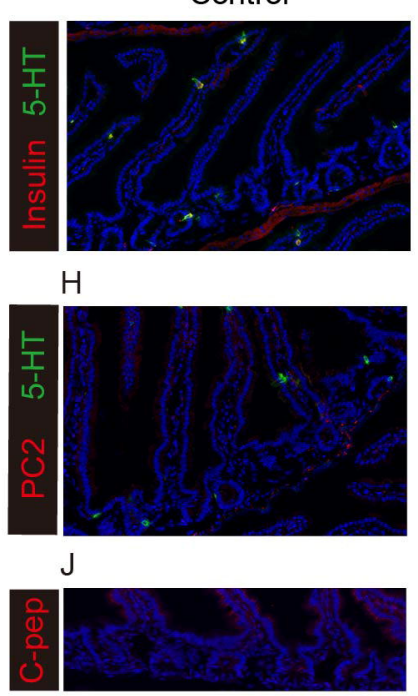

L

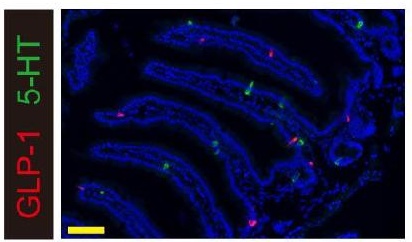

B

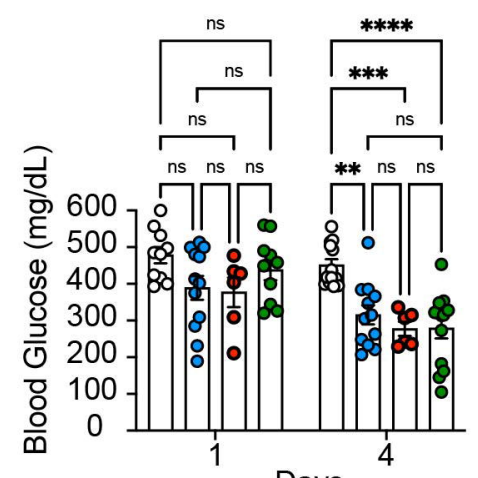

Days

E
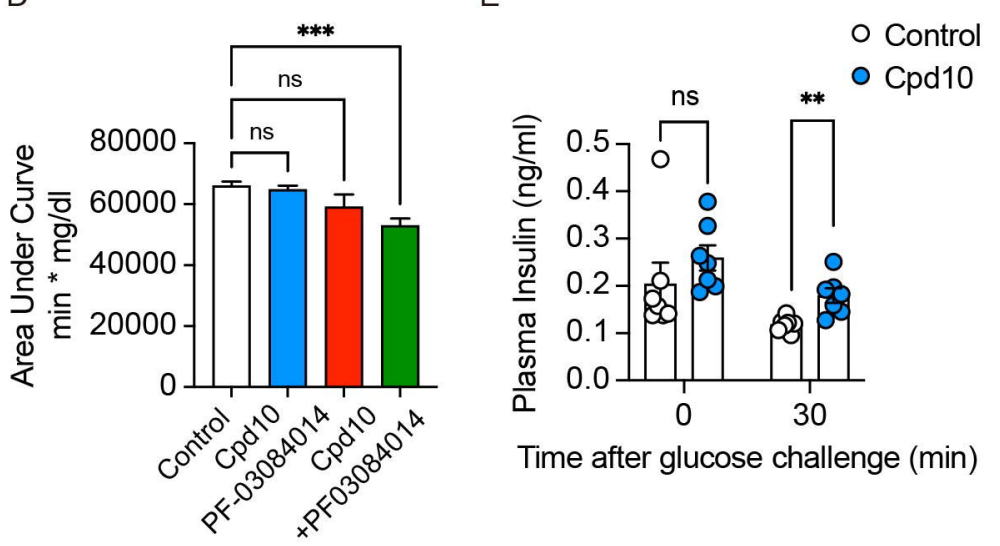

N

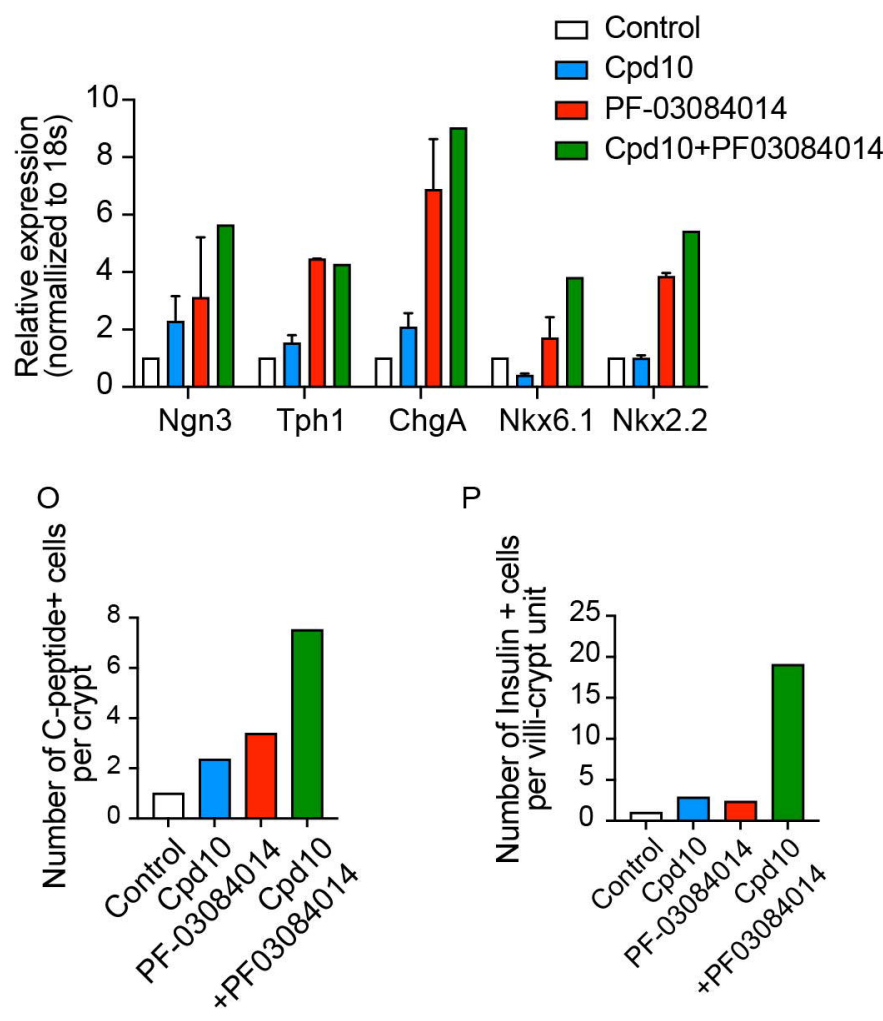

